Article

\title{
Modeling and Detection of Future Cyber-Enabled DSM Data Attacks
}

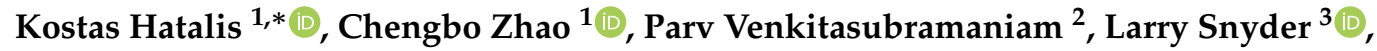 \\ Shalinee Kishore ${ }^{2}$ and Rick S. Blum ${ }^{2}$ \\ 1 Department of Electrical and Computer Engineering, Lehigh University, Bethlehem, PA 18015, USA; \\ chz317@lehigh.edu \\ 2 Faculty of Electrical and Computer Engineering, Lehigh University, Bethlehem, PA 18015, USA; \\ pav309@lehigh.edu (P.V.); shk2@lehigh.edu (S.K.); rblum@lehigh.edu (R.S.B.) \\ 3 Faculty of Industrial and Systems Engineering, Lehigh University, Bethlehem, PA 18015, USA; \\ larry.snyder@lehigh.edu \\ * Correspondence: kmh511@lehigh.edu
}

Received: 16 July2020; Accepted: 17 August 2020; Published: 21 August 2020

\begin{abstract}
Demand-Side Management (DSM) is an essential tool to ensure power system reliability and stability. In future smart grids, certain portions of a customer's load usage could be under the automatic control of a cyber-enabled DSM program, which selectively schedules loads as a function of electricity prices to improve power balance and grid stability. In this scenario, the security of DSM cyberinfrastructure will be critical as advanced metering infrastructure and communication systems are susceptible to cyber-attacks. Such attacks, in the form of false data injections, can manipulate customer load profiles and cause metering chaos and energy losses in the grid. The feedback mechanism between load management on the consumer side and dynamic price schemes employed by independent system operators can further exacerbate attacks. To study how this feedback mechanism may worsen attacks in future cyber-enabled DSM programs, we propose a novel mathematical framework for (i) modeling the nonlinear relationship between load management and real-time pricing, (ii) simulating residential load data and prices, (iii) creating cyber-attacks, and (iv) detecting said attacks. In this framework, we first develop time-series forecasts to model load demand and use them as inputs to an elasticity model for the price-demand relationship in the DSM loop. This work then investigates the behavior of such a feedback loop under intentional cyber-attacks. We simulate and examine load-price data under different DSM-participation levels with three types of random additive attacks: ramp, sudden, and point attacks. We conduct two investigations for the detection of DSM attacks. The first studies a supervised learning approach, with various classification models, and the second studies the performance of parametric and nonparametric change point detectors. Results conclude that higher amounts of DSM participation can exacerbate ramp and sudden attacks leading to better detection of such attacks, especially with supervised learning classifiers. We also find that nonparametric detection outperforms parametric for smaller user pools, and random point attacks are the hardest to detect with any method.
\end{abstract}

Keywords: demand side management; demand response; cyber-physical systems; dynamic pricing; load forecasting; attack detection

\section{Introduction}

Demand-Side Management (DSM) is an essential component in smart grids for planning, monitoring, and modifying consumer load levels. Furthermore, cyber-enabled DSM will allow smart grids even higher levels of automated decision-making capabilities to selectively schedule loads on 
local grids to improve power balance and grid stability. Such a cyber approach relies heavily on real-time, two-way communication capabilities between a central controller and various adaptable loads. Research into the reliability of the cyberinfrastructure that enables DSM is therefore vital. The main concerns in ensuring DSM security lay in the feedback mechanism of real-time electricity pricing and distributed DSM controllable loads. For instance, in residential grids, a single home consumes only a small amount of electric power, and if it becomes compromised, then it might not have a noticeable impact on the power grid. However, a carefully planned or even random cyber attack might impact other loads not under attack by taking advantage of the feedback mechanism of load management.

Two-way communication capabilities of Advanced Metering Infrastructure (AMI) enable a utility or Independent System Operator (ISO), in the retail power markets, to collect high-resolution energy usage from consumers and enable dynamic pricing to adapt to consumer demand [1]. AMIs provide an efficient way for ISOs to schedule prices and then communicate them to consumers for automatic DSM control of certain portions of a consumer's load. AMIs can also provide practical ways for ISOs to set DSM goals, such as reducing peak or decreasing aggregate load levels through price influences. However, there are several vulnerabilities in AMIs that present noteworthy security issues since they are directly accessible by users [2]. Due to the large scale deployments of AMIs, ISOs encourage the utilization of marginally cheaper hardware, resulting in constrained computational resources to allow for robust security capacities, such as intrusion monitoring.

DSM programs also utilize demand response, a specific tariff or program to motivate customers to respond to changes in price or electricity availability over time by altering their regular electricity use habits. We take this a step further and look at future cyber-enabled DSM programs [3] that will autonomously control household loads such as water heaters and HVAC units based on Real Time Pricing (RTP). As part of the reliable implementation of this future cyber-DSM, it is crucial to understand the dependency between dynamic pricing and automatic demand response as well as the risks. We hypothesize that cyber-DSM programs can be particularly vulnerable to cyber attacks such as false pricing information or direct load manipulation, especially when the participation rate in DSM is high.

Our work is thus motivated to study these vulnerabilities in DSM. We provide a mathematical formulation of the feedback between utilities and DSM systems, and then simulate, analyze, and test different detection methods for attacks on such feedback. This relationship between load and price is shown visually in Figure 1. As prices go up, demand naturally responds by decreasing. However, if AMIs are hijacked, and false lower prices are reported to DSM systems, there will be an inappropriate increase in demand. A similar effect happens if an attacker directly controls user loads. Then, a higher load usage by the attacker may inadvertently lead to higher prices for the rest of the grid. We present a standard of how attackers can exploit such a dependency.

We propose a mathematical framework of the feedback between price setting and DSM systems to study how attacks can be structured and how to detect them. Similar frameworks have been considered in previous work. In [4], the author applied a profitable attacking strategy to a price-load dependency model. Another control-theoretic approach to derive the fundamental conditions of Real Rime Pricing (RTP) stability under integrity attacks is described in [5]. The authors in that work use an elasticity model between price and load and apply an autoregressive model to obtain the forecast load and price. The main contributions of our approach can be summarized as follows:

1. We formalize the demand-price relationship using a nonlinear elastic demand model to achieve DSM goals. We simulate load and pricing data in a DSM system, in particular under a strategic conservation scheme.

2. We propose two modes of attacks on DSM systems: false pricing data injection and direct load manipulation. We prove their equivalence and highlight three types of attacks that could be undertaken by each mode. We then empirically show how an increased use of DSM can exacerbate attacks. 
3. We simulate these attacks and compare sequential change-point and machine learning methods, as well as a deeper dive in parametric vs. nonparametric methods, for detecting DSM attacks. In our main results, we demonstrate the impact of DSM on detection performance and identify what kind of detectors are effective for different attacks.

In Section 2, we provide a literature review on DSM and important DSM strategies on real-time pricing and load forecasting. In Section 3, we present a block bootstrap technique for simulating the non-DSM load distribution of a micro-grid of $N$ homes from template residential load time series. We then propose dependency models for the feedback nature of load and prices in Section 4, where we also showcase simulations of residential load and electricity prices when an automatic DSM program controls certain portions of consumer demand as a function of price. In Section 5, we present two modes of cyber attacks, direct load manipulation attacks, and price data injection attacks that can have a significant influence on the feedback of load and price. We prove these two attacks are equivalent. In Section 6, we review several sequential detection and supervised learning methods. In Section 7, we present our experimental results that compare performance under different DSM participation rates when they are under three types of attacks: ramp, sudden, and point attacks. We find that point attacks are hardest to detect, and supervised learning methods can provide better results than sequential detection methods. To provide a deeper analysis, we also conduct a comparison of parametric and nonparametric detectors. We conclude in Section 8 with possible directions of future work.

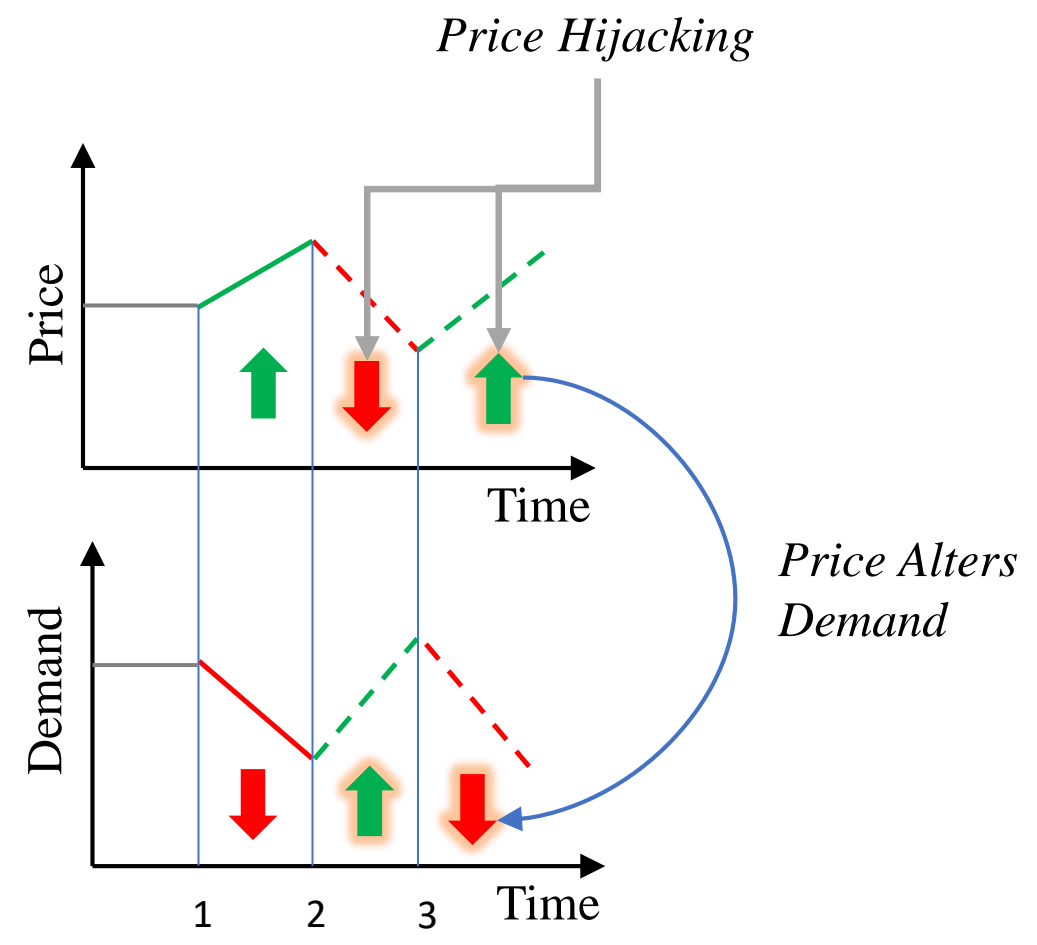

Figure 1. Feedback effect between price and DSM demand. As prices go up, demand decreases. However, if prices are hijacked and false prices are fed to DSM systems, then a false low price increases demand, and a false high price can decrease demand. The same is true if demand was altered by an attack. If load usage is increased by an attacker, then prices would increase and vice versa. 


\section{Background}

\subsection{Demand Side Management}

DSM is an active and voluntary approach for reducing electricity use through activities or programs that promote electric energy efficiency, conservation, or more efficient management of electric energy loads [6]. Very often, ISO's utilize financial incentives and educational programs to modify consumer demand. The main goals of DSM are peak clipping, valley filling, load shifting, strategic load growth, flexible load shaping, and strategic conservation. We summarize these goals in Figure 2. In these goals, consumers are encouraged to use less energy during peak hours, or to move the time of energy use to off-peak times such as nighttime, or reduce overall consumption. Other applications for DSM are to aid grid operators in balancing intermittent generation from wind and solar farms due to their volatile nature, which may not coincide with energy demand at different times.

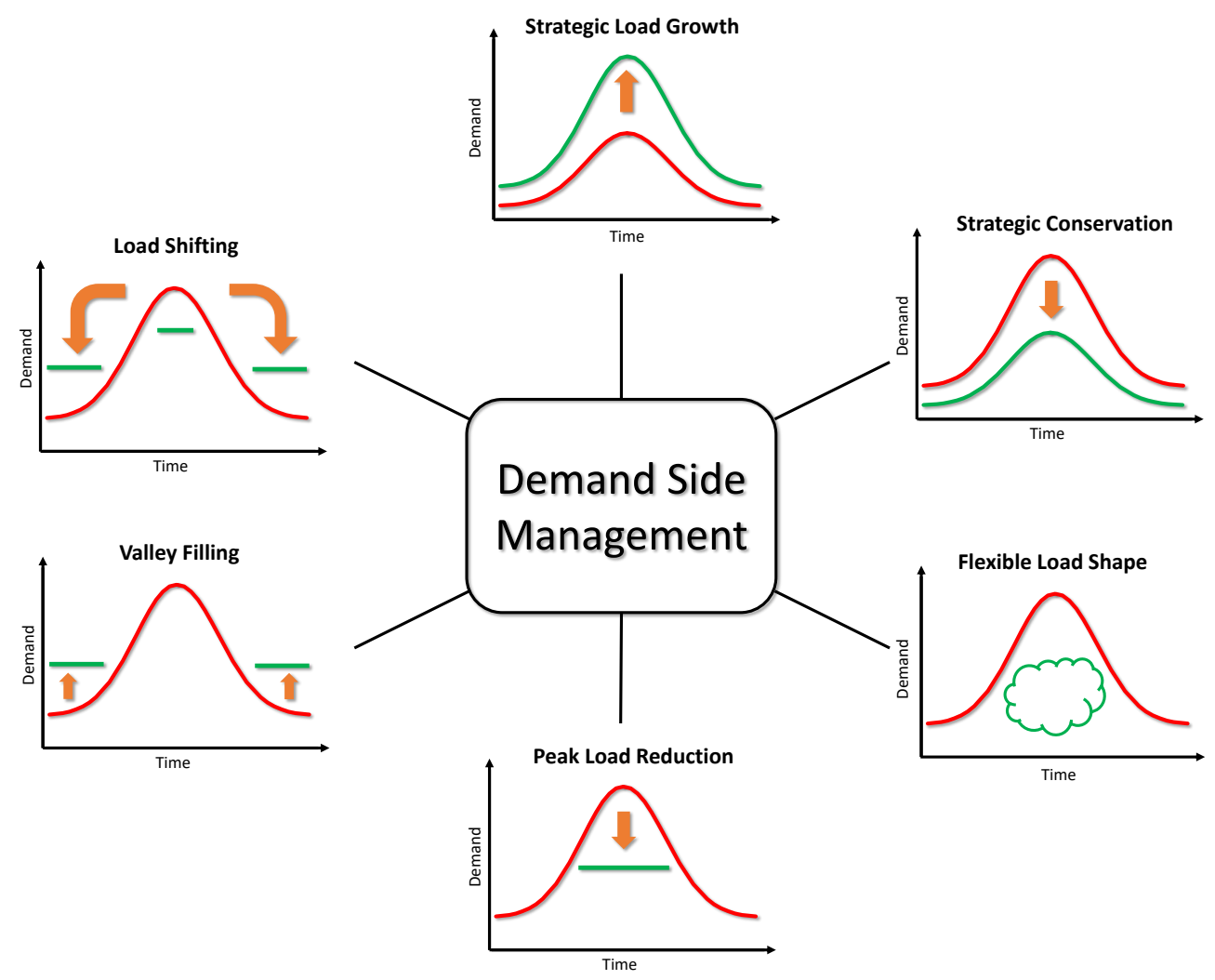

Figure 2. Various demand side management goals.

Our study focuses on modeling and simulating the DSM goal of strategic conservation due to its simplicity and essential use in the smart grid. This goal also makes it easier to study attacks on DSM by modeling strategic conservation as a general reduction in load. Attacks then could stand out more visibly than under other goal schemes like flexible load shaping. More specifically, this DSM goal aims at reducing aggregate load demand through directed reduction of electricity consumption. The successful implementation of strategic conservation programs usually requires some combination of financial incentives to customers, energy-efficient building standards, and appliance efficiency improvement. We envision that AMI and smart appliances in residential DSM programs will automatically control specific portions of consumer load as a function of real-time electricity prices to achieve the goal of strategic conservation. 
Most DSM programs are formulated as an optimization problem as follows:

$$
\min _{P_{t}} \sum_{t=1}^{T}\left(L_{t}-L_{t}^{\prime}\right)^{2}
$$

where $P_{t}$ is the RTP at time $t, L_{t}$ is actual load, and $L_{t}^{\prime}$ is the target load level the ISO is interested in achieving via DSM. The aim is to choose a price $P_{t}$ for each time step such that the actual load would reach as close as possible to the target level. In [7], a DSM strategy is proposed based on a heuristic optimization strategy to shape the load curve close to the desired shape. A heuristic-based evolutionary algorithm was used to solve the above minimization problem. A multi-agent game-theoretical DSM approach is proposed in [8]. The authors use game theory and formulate an energy consumption scheduling game, where the players are the users, and their strategies are the daily schedules of their household appliances and loads. In [9], the minimization problem is solved by utilizing a feedforward neural network to map the nonlinear relationship between price and load. Recently, the DSM problem is addressed in [10] as a multi-objective optimization problem that also seeks to balance other merit functions such as energy production cost, customer preferences, and other constraints.

\subsection{Load Forecasting}

Load Forecasting (LF) techniques are essential for RTP and other ISO operations by predicting future energy requirements of a system from previous data and weather conditions. It is recognized as the initial building block of utility planning efforts and ensures the balance between supply and demand of energy; LF thus plays a vital role in our DSM formulations. For a given system and requirements, LF provides predictions for specific periods. These periods are divided into short-, medium-, and long-term forecasts. Short-term LF is used to predict the load on an hourly basis for up to one week when considering daily operations and cost minimization. Medium-term LF typically predicts load on a weekly, monthly, or yearly basis for efficient operational preparations. Long-term LF is used to predict the decades' load to facilitate grid and generation expansion planning. In this work, we look at short-term LF to predict loads from one hour to a week.

LF models can be divided into two approaches [3]: the first being statistical based modeling and the second being machine learning. The statistical approach can be further broken down into regression and time series models. Multiple linear regression can be used with the weighted least squares estimation technique to form a relationship between different independent covariates when load depends on such things as weather conditions. Regression models have been applied in LF in different works, such as in [11]. Time series models are also prevalent to apply to LF. The most common model is the AutoRegressive Moving Average (ARMA) model and its variants that include components such as integration (I), Fractional Integration (FI), multiVariate series (V), Seasonality (S), eXogenous $(\mathrm{X})$ data, Conditional Heteroskedasticity $(\mathrm{CH})$, and Nonlinearity $(\mathrm{N})$. For our formulation, we employ SARIMA for LF.

Hyperparameters of ARMA models can be configured using Box-Jenkins decomposition or grid-search with the Akaike information criterion. Numerous studies have looked at all the different ARMA models for LF [12]. Other time series methods for LF include simple exponential smoothing [13] and the Holt-Winters seasonal method [14]. Time series analysis and regression analysis share many models and ideas, but they are theoretically different. Time series analysis first deals with time-indexed stationary data and accounts for the autocorrelation between time events. In regression, we assume there is no autocorrelation, and that all observations are independent and identically distributed. Furthermore, we also assume that in regression, the data are homeostatic and do not exhibit multicollinearity.

Most recently, machine learning methods have seen a huge spike in LF research. Machine learning models are data-driven, typically providing a nonlinear fit to input covariate data to predict load. The advantages of this approach include not needing preconditions for data such as stationarity 
(a requirement for most time series methods), excels at modeling nonlinear dependencies, and can fit large data sets. Disadvantages for most machine learning models are that most hyperparameters are continuous (difficult to tune), they require extensive feature engineering, and may get stuck in local minimums. Models for LF include support vector machines [15], feedforward neural networks [16], recurrent neural networks [17], random forests [18], and ensemble learning [19].

\subsection{Real-Time Pricing}

Every consumer of electricity is charged a certain amount per kiloWatt-hour (kWh) of energy. Such a charge covers the costs associated with the generation, transmission, and distribution of electricity. The two main types of costs are operational and fixed costs. During the 20th century, tariffs were used to recover costs. Lately, clever pricing schemes have been developed to meet the requirements of modern power systems [20], such as RTP, where consumers are charged with a price closest to the real price of generation at a specific interval in time. RTP plays an integral part in time-based DSM programs that make consumers choose the time of consumption of power as a response to prices [21]. Cyber-enabled DSM programs are an automated form of time-based DSM programs.

There are two types of RTP schemes, hourly pricing, and day-ahead pricing. In the first type, the price of electricity is released on an hourly basis for the next hour. In day-ahead pricing, prices are announced for the next $24 \mathrm{~h}$ based on predicting the load demand and the cost of generation. RTP signals combined with DSM automation at the consumer level provide benefits to both consumers and utility. An adequately designed RTP scheme increases the grid's reliability, reduces associated costs with the generation, and lowers electricity bills of consumers. Further review of RTP and other dynamic pricing schemes can be found in [3].

\section{Microgrid Simulation}

Before modeling the load-price dependency of a DSM system, we first need to obtain some ground truth data of what load data from a residential micro-grid look like without the presence of DSM, where we assume the elasticity of demand to price is very low. To do this, we use the power time series from several homes as templates for our grid and generate artificial $N$ household datasets. There are several ways to generate artificially residential load data, such as using power grid simulators like MATPOWER [22] or GridLAB-D [23]. Our object is to create a model-free real-data driven alternative to grid simulators. We can generate unlimited but plausible univariate load data to serve as the base demand for sample households before applying a DSM system.

\subsection{Data Source}

We use the UMass Smart* [24] dataset, 2017 release, for the simulation of micro-grid load time series. The Smart* project built a data collection infrastructure that records data from a variety of sensors deployed in real homes. Their infrastructure supports both pulling data by querying individual sensors and pushing data from sensors to a gateway server, which ran on their software tools. The 2017 Home dataset release is comprised of electrical power readings from seven homes from 2014 to 2016 at a minute resolution. It includes readings from individual appliance sensors as well as total power usage of each home. We chose to use these seven datasets as template homes in simulating the power usage of a micro-grid due to the breadth of the data collected. For DSM attack research, these datasets can help model an attacker compromising individual appliances. For each home, the power consumption is given in $\mathrm{kW}$ for every minute. We convert this time series to $\mathrm{kWh}$ with a resolution of one hour, which is typical for smart meter readings and RTP modeling [3]. We do this by obtaining the average power consumption within an hour and multiplying it by the time period as such

$$
E_{(k W h)}=t_{(h r)} \times \frac{1}{60} \sum_{i=1}^{60} P_{(k W)}^{i}
$$




\subsection{Block Bootstrap Simulation}

In the generation of new time series from sample data, several approaches can be applied depending on the series' statistical properties. Data that are stationary can be modeled and generated using an ARMA process [25]. An ARMA model is fitted to the data, and then future data are sampled from the ARMA distribution. If there is no serial correlation, then the distribution of some sample data can be modeled using Markov Chain Monte Carlo [26], and new data can be sampled from this estimated distribution. However, in the case that data exhibited autocorrelation and non-stationarity in the presence of a periodic seasonal pattern, a natural choice is to use the block bootstrap method [27].

The bootstrap method comes from simulation statistics for estimating the distribution of a statistic such as the mean or variance. Bootstrap is particularly useful when there is no analytical form to estimate some underlying distribution. A bootstrap analysis is conducted by using the Monte Carlo algorithms with replacement. Data are sampled with replacement until a new set is formed, and then statistics are calculated from that new set. The process can be repeated to get a more precise estimate of the Bootstrap distribution and form confidence intervals for those statistics. The block bootstrap is used when the data, or the errors in a model, are correlated. The block bootstrap attempts to replicate the serial correlation by resampling blocks of data instead of individual observations. This is why the block bootstrap is used primarily with correlated time series. In block bootstrap, blocks sampled can overlap or be non-overlapping. For load time series simulation, we use block bootstrap with non-overlapping blocks to preserve the daily seasonal pattern of power consumption. The process of block bootstrap simulation of a new home power usage from a template home is shown in Figure 3.
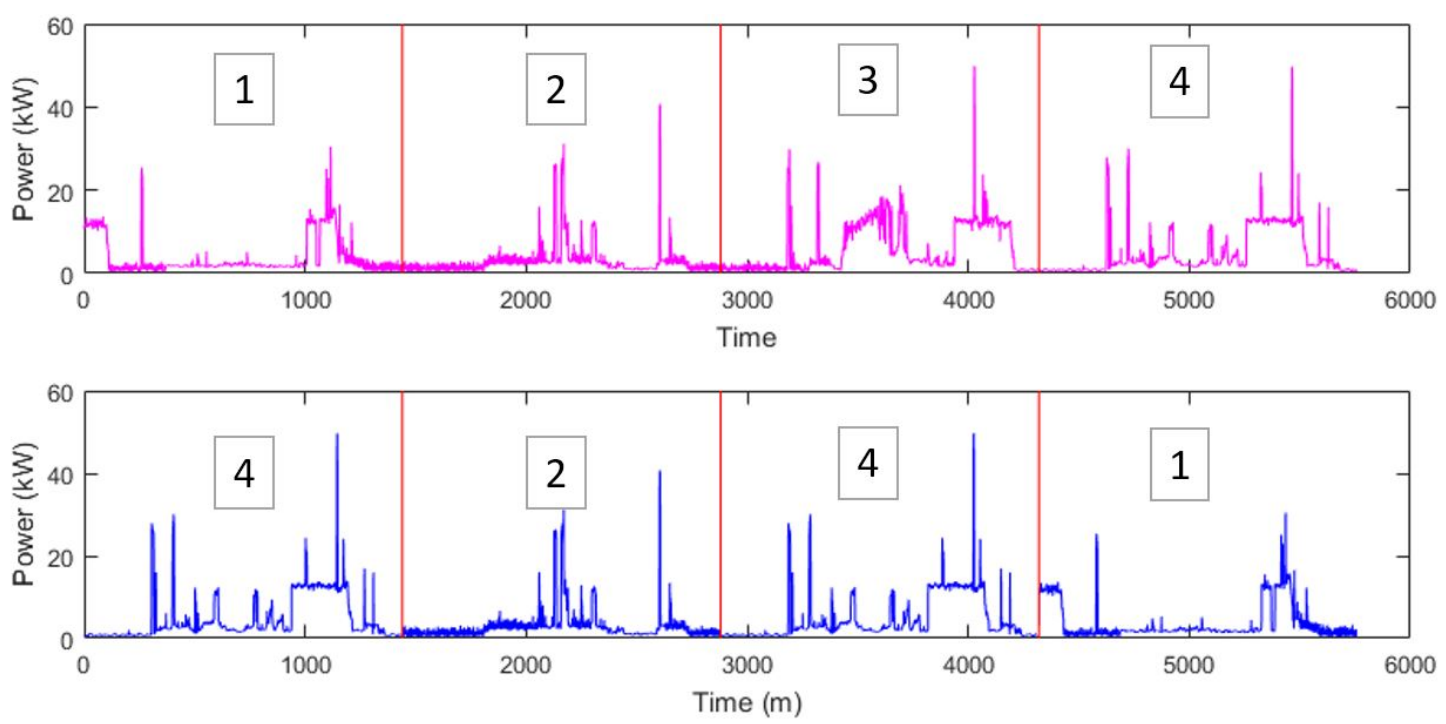

Figure 3. Process of block bootstrap simulation of a new home power usage (bottom) from a template home (top). Example simulation samples are taken from four days from the template series with replacement.

\section{Dependency Model}

\subsection{Modeling Elastic Demand}

For analyzing the feedback dependency between load and price in a DSM setting, we first need to define the supply and demand relationship of electricity. To do so, we utilize the well-known measure in economics, the Price Elasticity of Demand (PED) [28], which can be given by

$$
\epsilon_{d}=\frac{d L}{d P} \cdot \frac{P}{L}
$$


PED shows the responsiveness of the load $L$ demanded of electricity to a change in Price (P). Recent work [9] in demand response, particularly in the context of cybersecurity, has utilized this approach. Our procedure differs in that we use real demand data to determine the "unmodulated" pattern of load data. An absolute value of PED $=1$ shows unitary elasticity. For instance, when $\epsilon_{d}=-1$, then a $1 \%$ change in the price will have a $1 \%$ change in the load demanded. As prices increase, the load will decrease. When absolute PED falls between 0 and 1 , this signifies that the load demand is inelastic, while a value greater than 1 says that the demand is elastic. When $\left|\epsilon_{d}\right|=0$, the demand is perfectly inelastic. A change in price does not affect the load, while $\epsilon_{d}=\infty$ represents perfect elasticity. If $\epsilon_{d}$ is constant, the whole demand curve is then

$$
L=a \cdot P^{\epsilon_{d}}
$$

where $a$ is a scaling constant. An example demand curve estimated from Equation (3) can be seen in Figure 4 . The figure also showcases the nonlinear relationship between load and price where as the price, the independent variable, increases, the load demanded, the dependent variable, decreases.

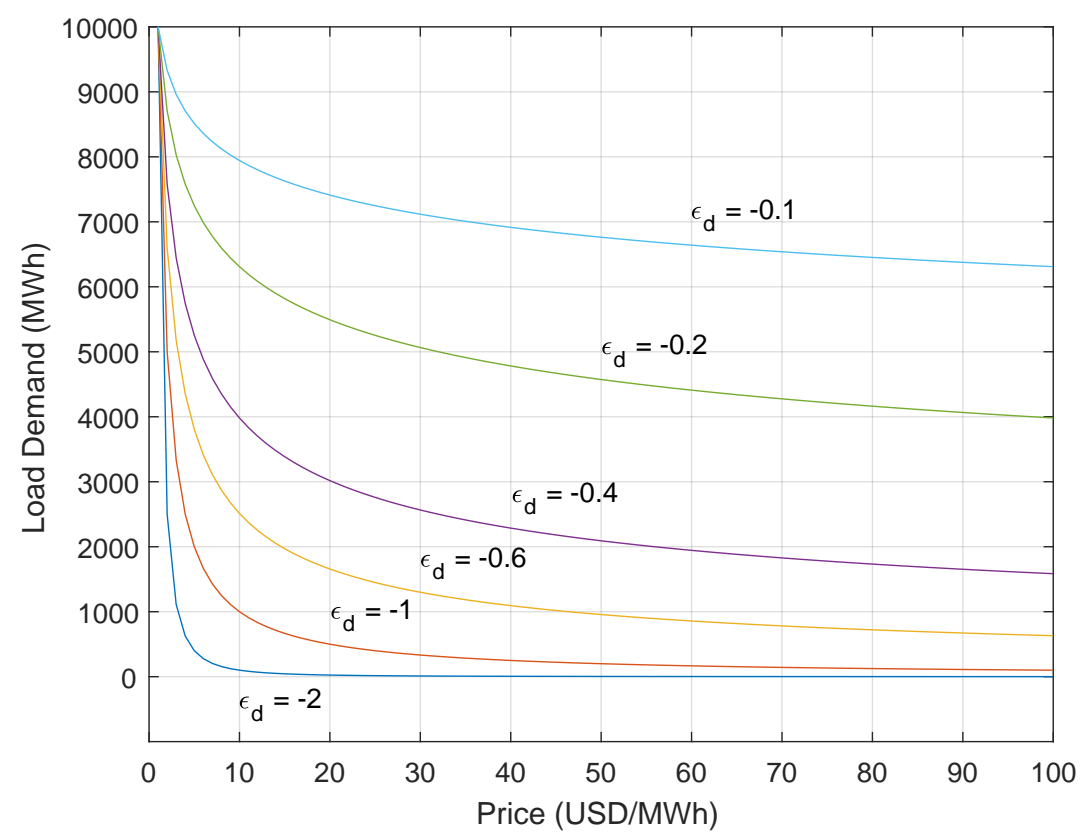

Figure 4. Load as a function of price with arbitrary price range $\$ 1-\$ 100, \alpha=10,000$, and $\epsilon_{d}=$ $-0.1,-0.2,-0.4,-0.6,-1,-2$.

\subsection{Modeling Consumer DSM}

In modeling, the relationship between load and price under a DSM program, it is important to define each customer's individual loads in determining the aggregate load. For a customer who does not participate in a DSM program, their load is determined by the stochastic demand process of users' actions such as watching TV or using the AC. Demand is impacted by multiple factors such as user preferences, weather, and time of day. In this process, electricity prices have a small influence on demand; individual customer demand is fairly inelastic to price. Following the derivation in Equation (3), we define the load usage of an individual customer $i$ for time $t$ as

$$
\phi_{t, i}=\theta_{t, i}\left(P_{t}+P_{c}\right)^{\epsilon_{t, i}^{d}}
$$

where $P_{t}$ is the RTP for time $t, P_{c}$ is constant of the retailer's market costs which does not vary with RTP, $\theta_{t, i} \geq 0$ is a scaling factor representing the stochastic process that determines the user load, and $\epsilon_{t, i}^{d}$ is 
the elasticity coefficient for the individual customers sensitivity to price changes. It can vary over time but, without DSM incentives, most users have a fairly inelastic PED.

For experimental purposes, in modeling individual user loads, we set $\phi_{t, i}$ equal to simulated bootstrapped user load profiles defined in Section 3.2. We assume that prices, user preferences $\theta_{t, i}$, and $\epsilon_{t, i}^{d}$ have been absorbed in the calculation of the simulated load series. Thus, we use $\phi_{t, i}$ as a reference point to show how much electricity a user wants to consume without the influence of a DSM program. We model the task of the demand response administrator as modifying $\phi_{t, i}$ to some desired load levels.

Realistically, only a certain portion $\kappa_{t, i} \in[0,1)$ of customer $i$ 's power usage will be under control of a cyber DSM program. There will always be some stochastic and, more importantly, an inelastic component of power usages, such as using a microwave oven or electric hairdryer. We model the DSM modulated load (including the inelastic portion) as follows:

$$
l_{t, i}=\left(\kappa_{t, i} \phi_{t, i}\right) P_{t}^{\epsilon_{t, i}^{d s m}}+\left(1-\kappa_{t, i}\right) \phi_{t, i}
$$

where the first part $\left(\kappa_{t, i} \phi_{t, i}\right) P_{t}^{\epsilon_{t, i}^{d s m}}$ is the load level customer $i$ that allows the DSM program to determine as a function of price $P_{t}$ and the DSM's elasticity to price $\epsilon_{t, i}^{d s m}$. Price elasticity of DSM $\epsilon_{t, i}^{d s m}$ may vary over time and customer and affects how much power usage should be affected by price. The second portion $\left(1-\kappa_{t, i}\right) \phi_{t, i}$ of a customers load is the stochastic component. The DSM portion parameter $\kappa_{t, i}$ is defined as a function of time where users can add or remove house loads under DSM control over time. The term $\kappa_{t, i}$ can be modeled as a random variable (e.g., Uniform or Gaussian) or as a fixed constant for users. Once each customer's load is determined, total load (modified by a cyber-DSM program) for time $t$ for $N$ customers is calculated by

$$
L_{t}=\sum_{i=1}^{N} l_{t, i}
$$

We also define the aggregate base load as

$$
\Phi_{t}=\sum_{i=1}^{N} \phi_{t, i}
$$

which represents the total demand had there not been a DSM program for a time period $t\left(\operatorname{IE} \kappa_{t, i}=0, \forall_{i}\right)$.

\subsection{Modeling Strategic Load Conservation}

We propose the following approach to model the real-time pricing from a strategic load conservation perspective, wherein, on an hourly basis, the price is a function of the aggregate load to achieve the desired load level $L_{t}^{\prime}$. The approach takes as input a forecast $\hat{\Phi}_{t}$ of the base aggregate load to calculate price $P_{t}$. This prediction can be defined as

$$
\hat{\Phi}_{t}=f_{\text {pred }}\left(\Phi_{t-k: t-1}, P_{t-k: t-1}, X\right),
$$

where inputs to the prediction model are past base load $\Phi$ and price $P$ values from time $t-k$ to $t-1$, and other predictor variables $X$ such as time-of-day and weather information. Various types of 
prediction models can be used for $f_{\text {pred }}$ such as neural networks as reviewed in Section 2.2. We now define RTP based on the formulation in Equation (3) as such

$$
\begin{aligned}
& P_{t}=\left(\frac{L^{*}}{\hat{\Phi}_{t}}\right)^{1 / \hat{\epsilon}^{d s m}} . \\
& L^{*}=a \cdot P_{t}^{\hat{\epsilon}^{d s m}}
\end{aligned}
$$

where we can have two goal scenarios for $L^{*}$

$$
\begin{aligned}
& \text { Goal 1: } L^{*}=L_{t}^{\prime} \\
& \text { Goal 2: } L^{*}=L_{t}^{\prime}+\left(L_{t-1}^{\prime}-L_{t-1}\right)
\end{aligned}
$$

The component $L^{*}$ adjusts the RTP based on two goals the ISO may have. The first goal is to adjust the price to push power usage directly to the target level $L_{t}^{\prime}$ with the assumption that there is near $100 \%$ DSM participation by all customers. The expectation is that, if demand for time $t$ is $\hat{\Phi}_{t}$, then a price point is set to push load usage to $L_{t}^{\prime}$. Of course, if participation is less than $100 \%$, which is more likely, the target level $L_{t}^{\prime}$ will not be reached by $P_{t}$. To push the aggregate load from all users, as close as possible to the target load level with an unknown amount of participation, a penalty would need to be added to $P_{t}$. We model this as Goal 2, where the idea is to affect the power usage of those under cyber-DSM control even more than goal 1 to compensate users who are not participating in DSM.

Some users will not be participating, or only have a small portion of their power usage under control by the cyber-DSM program. We model their remaining power usage as inelastic to RTP. Thus, to push aggregate load to a target level, taking into account that some load usage is inelastic, we need to push RTP much higher or lower to have a bigger effect in pushing DSM controlled load closer to the target load. This is what Goal 2 attempts to do, with the component $L^{*}=L_{t}^{\prime}+\left(L_{t-1}^{\prime}-L_{t-1}\right)$ taking the target load level for time $t$ and adding the difference from the previous target load $L_{t-1}^{\prime}$ and realized load $L_{t-1}$ as a penalty to adjust RTP to compensate for the difference. If $L^{*}<0$, then we set $L^{*}=10$ or to some arbitrary small target value. By subtracting the difference between the previous load and target level, we make up for users not participating in DSM by forcing DSM users to have a higher price to push their load even lower.

The term $\hat{\epsilon}^{d s m}$ in Equation (8) is an estimate of the price elasticity of DSM of the whole grid; if individual user coefficients $\epsilon_{t, i}^{d s m}$ are unknown, then $\hat{\epsilon}^{d s m}$ can be estimated from observing past values of price and load under different levels of DSM control. Alternatively, the ISO can define $\epsilon^{d s m}$ for all household cyber DSM programs. The formulation in Equation (8) sets prices by comparing the adjusted target load for time $t$ to the forecasted base demand $\hat{\Phi}_{t}$ for the same time. This demand $\hat{\Phi}_{t}$ would be the level if no load were under DSM influence, thus to influence and alter it, $\Phi_{t}$ needs to be estimated as accurately as possible.

In our approach, if the aggregate load is above the target load, RTP is set higher to decrease demand. If the aggregate load is lower than the target load, the price decreases to increase demand. Thus, as also can be observed in Equation (8), there is direct feedback between price and load Equation (4). The block diagram in Figure 5 also outlines this feedback that showcases the relationship between the utility and the grid. Generation sets the target load based on the price and supply of power, and the controller sets the price signal and the elasticity of demand coefficient for DSM systems. The price is then communicated into the grid into DSM systems, which adjust load usage appropriately. The bold red lines in Figure 5 highlight the feedback relationship between price and demand. The scope of work is in the mathematical modeling of the controller and DSM system relationship. With such a model, we present in the next section how attackers can exploit it. 


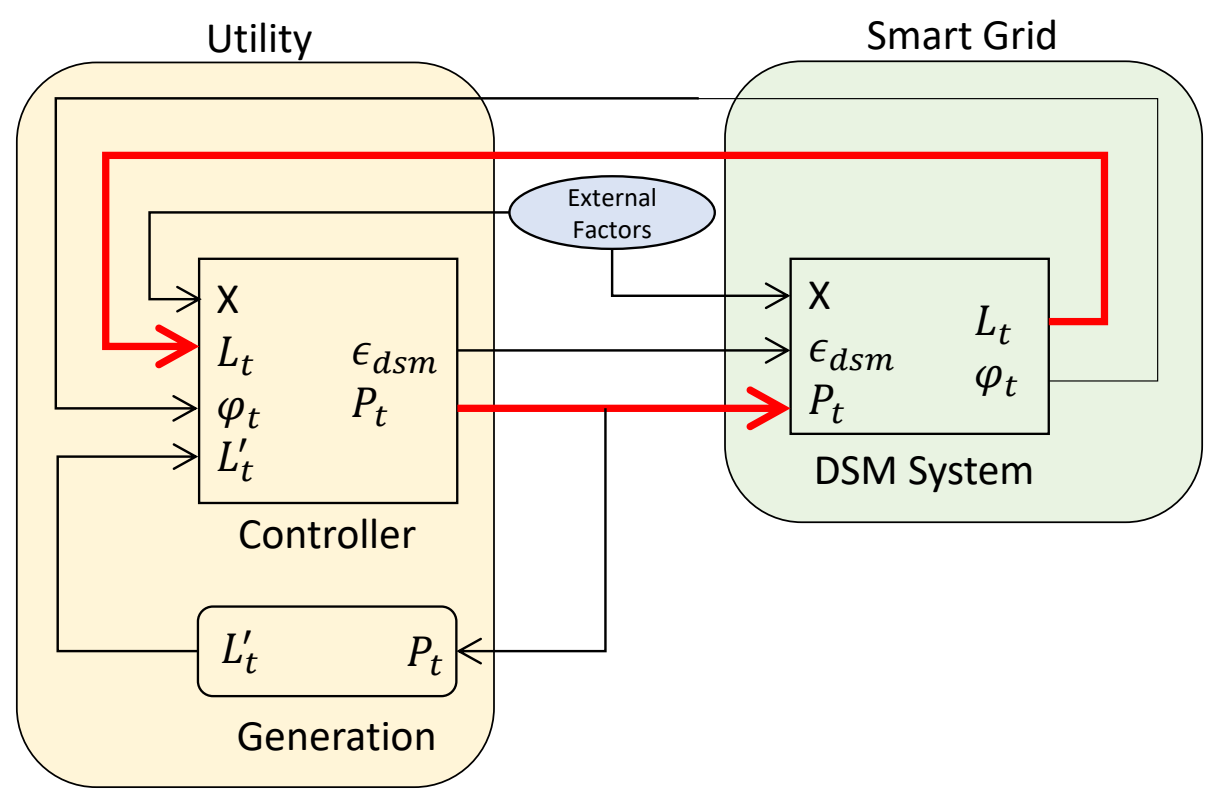

Figure 5. Block diagram highlighting the feedback between the utility and grid.

\subsection{Simulation Parameters and Assumptions}

For experimental purposes, in modeling individual user loads, we set $\phi_{t, i}$ equal to simulated bootstrapped user load profiles defined in Section 3.2. We assume that prices, user preferences $\theta_{t, i}$, and $\epsilon_{t, i}^{d}$ have been absorbed in the calculation of the simulated load series. Thus, we use $\phi_{t, i}$ as a reference point to how much electricity a user wants to consume without the influence of a DSM program. We model the task of the ISO as modifying $\phi_{t, i}$ to some desired load levels. Furthermore, we include the following assumptions in our modeling and simulation. We assume that the ISO can define $\epsilon^{d s m}$ for each household and we set it as a constant for all customers and time. For additional simplicity, we model $\kappa_{i}$ also invariant in time, but it may vary per customer. Lastly, through AMI, we assume the ISO can obtain an estimated reading of $\Phi_{t-1}$ but not of individual user $\phi_{t-1, i}$ to preserve privacy. This way the ISO has a time series of estimated non-DSM load demand in order to provide predictions $\hat{\Phi}_{t}$.

For all case studies in the rest of our paper, we use the UMass Smart* dataset (2017 release) to bootstrap simulate residential load as described in Section 3. We simulate a micro-grid of $N=200$ residential homes for a time period of $T$ to obtain a phi distribution that defines a base load profile for each home $i$ at each time $t$. We set the DSM demand elasticity for each user to $\epsilon^{d s m}=--1, \forall_{i}$ to allow the DSM component of customer load to be sensitive enough to price changes. For all case studies, we set a simple flat target load of $L_{\text {target }}=200 \mathrm{kWh}$. We note, however, that, with our pricing formulation in Equation (8), we can model any DSM goal from peak load reduction to flexible load shaping. These simulation parameters are summarized in Table 1. For forecasting $\hat{\Phi}_{t}$, we use the naive persistence prediction method.

Table 1. Simulation parameters used in case studies.

\begin{tabular}{ll}
\hline \multicolumn{2}{l}{ Simulation Parameters } \\
\hline$N$ & 200 homes \\
$\epsilon_{d s m}$ & -1 \\
$L_{\text {target }}$ & $200 \mathrm{kWh}$ \\
\hline
\end{tabular}

In Figures 6 and 7, we demonstrate a price-load feedback simulation for a period of $T=48 \mathrm{~h}$ under Goals 1 and 2 with parameters defined in Table 1 . Under the Goal 1 scenario when $\kappa_{i}=0, \forall_{i}$, 
shown in Figure 6a, we see that prices range from $\$ 0.01$ to $\$ 0.03$ per $\mathrm{kWh}$. As expected with no DSM program to influence demand, the observed load is equal to the base load. The same is observed in Figure 7a when the simulation is run with the Goal 2 RTP model. The only difference under this scenario is the price range which is exceptionally high ranging from $\$ 0.01$ to $\$ 0.6$ per $\mathrm{kWh}$. This is expected in this scenario since the ISO is attempting to set prices to maximize the effect on DSM customers, of which there are none, but this is unknown to the utility. With no DSM, the mean observed load is $332 \mathrm{kWh}$.
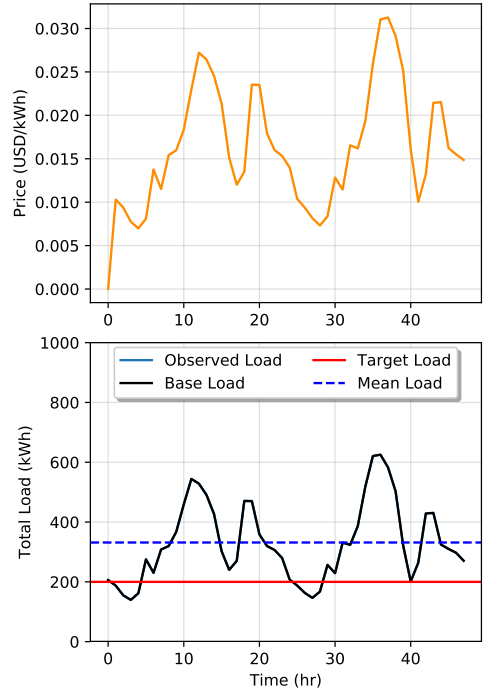

(a)
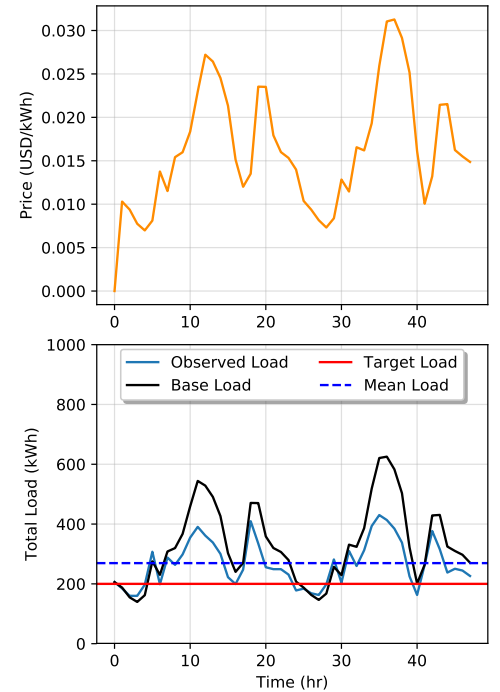

(b)
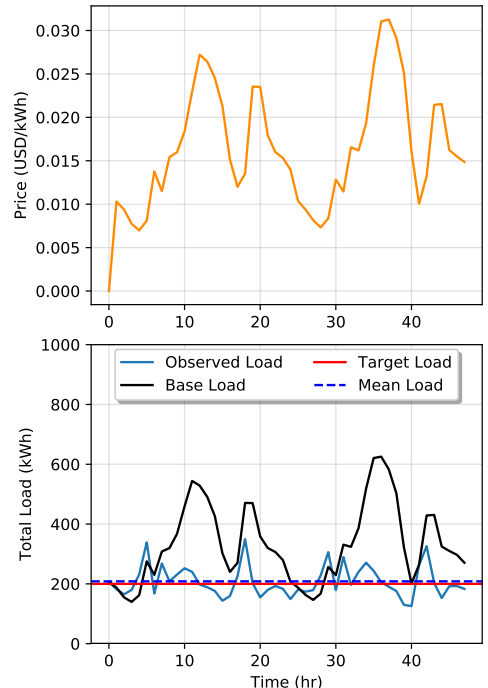

(c)

Figure 6. Simulation of price-load interaction with Goal 1 with DSM when $\kappa_{i}=0, \forall_{i}(\mathbf{a}), \kappa_{i}=0.5, \forall_{i}(\mathbf{b})$, and $\kappa_{i}=0.99, \forall_{i}$ in (c). Since this simulation is with Goal 1 , we see that, while price affects the load demand, load doesn't affect price.
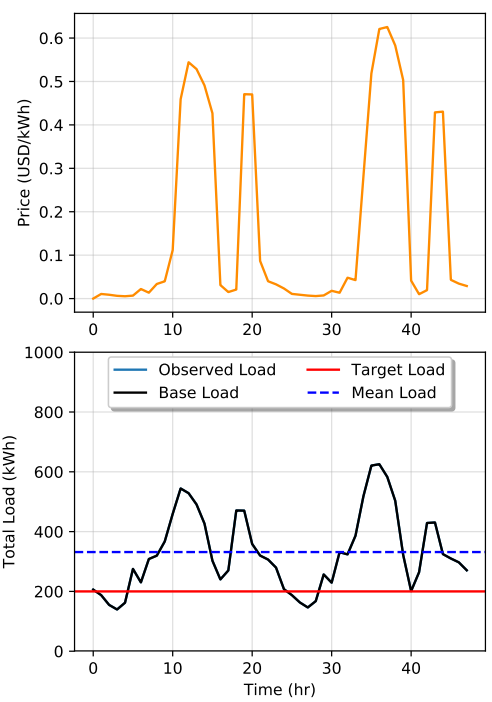

(a)
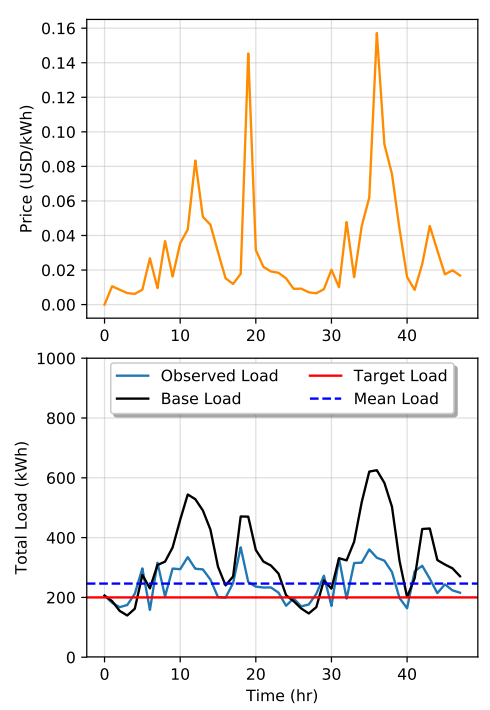

(b)
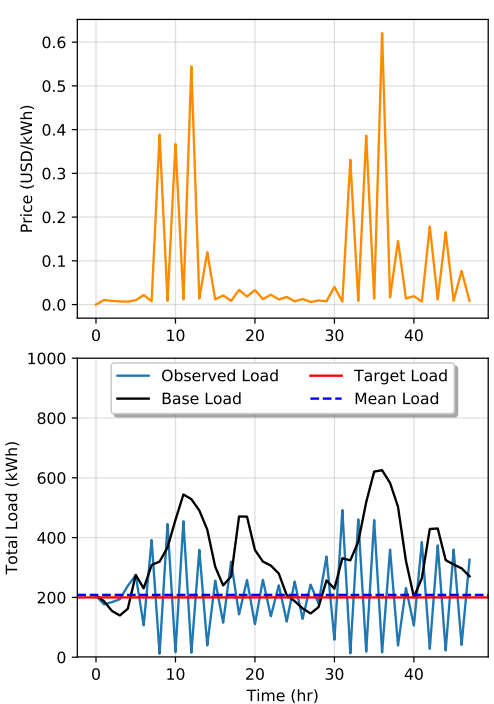

(c)

Figure 7. Simulation of price-load interaction with Goal 2 with DSM when $\kappa_{i}=0, \forall_{i}(\mathbf{a}), \kappa_{i}=0.5, \forall_{i}(\mathbf{b})$, and $\kappa_{i}=0.99, \forall_{i}$ in (c). In this simulation under with Goal 2, we see that price does affect the load demand and vice versa.

Next, we rerun the simulation setting $\kappa_{i}=0.5, \forall_{i}$. In the Goal 1 scenario in Figure $6 \mathrm{~b}$, we see that the base load was reduced with a mean observed load of $269 \mathrm{kWh}$. In Figure $7 \mathrm{~b}$, the same simulation 
is run with Goal 2. Here, the mean observed load was further reduced to $246 \mathrm{kWh}$, but large price spikes occur at peak load times. Finally, we run simulations for $\kappa_{i}=0.99, \forall_{i}$ to study the effects of high penetration of DSM. In simulating both goals, shown in Figures $6 \mathrm{c}$ and $7 c$, the mean observed load is reduced to $208 \mathrm{kWh}$, which is very close to the target load. However, in Goal 2, we see great resonating feedback effects occur when prices spike very high. RTP increases as a response to large values in the observed load. Then, when the load decreases to low levels, a price decrease causes the load to spike more during the next time step, while under Goal 1, this is not observed. The higher prices set in Goal 2 would see a large cost to DSM participating customers.

\section{DSM Attack Models}

An attacker can exploit the feedback between the customer and utility in determining RTP and load usage by cyber DSM programs by injecting false price or corrupted load data into the feedback loop. The attack exploitations we study here differ from the false data injection attacks studied in other smart grid papers. Most false data injection attack works [29] study the compromise in energy management systems to alter power state estimates by the utility operator. In our case, we study attacks that aim to alter a user's load profile by exploiting cyber DSM vulnerabilities.

For modeling attacks on a cyber DSM managed micro-grid, we assume that the attacker compromises a subset of all the $N$ customers; we denote this subset as $\mathcal{A}$, for an attack period $t \in T_{a}$. We study two modes of attacks: false pricing data injection attacks in which a compromised user receives manipulated pricing information and a direct load manipulation attack in which the compromised customer's appliances are under the control of the attacker. When communication encryption is broken with an AMI, then a pricing data injection attack can occur. A direct load manipulation can occur by hacking into a cyber DSM load controller, or directly hacking into smart appliances, altering a user's load profile. The two modes of attacks are outlined below.

- False Pricing Data Injection: The attacker can manipulate prices $P_{t}$ received by each compromised costumer $i \in \mathcal{A}$, and the received price $P_{t}^{i}$ can be different for various customers in order to achieve the attacker's desired effect:

$$
P_{t, i}^{a}=P_{t, i}+a_{t, i}^{P}, \forall_{i} \in \mathcal{A}, t \in T_{a}
$$

This has the effect of compromising the demand response of a customer in the following way:

$$
l_{t, i}^{a^{P}}=\left(\kappa_{i} \phi_{t, i}\right)\left(a_{t, i}^{P}\right)^{\epsilon^{d s m}}+\left(1-\kappa_{i}\right) \phi_{t, i}
$$

- Direct Load Manipulation: The attacker can manipulate the load of each compromised customer $l_{t, i}, i \in D$ directly:

$$
l_{t, i}^{a^{L}}=l_{t, i}+a_{t, i}^{L}, \forall_{i} \in \mathcal{A}, t \in T_{a}
$$

Under both attack modes, we would get a compromised aggregate load which may include one or both attacks occurring simultaneously

$$
L_{t}^{a}=\sum_{i=1}^{N} l_{t, i}^{P^{P}}+l_{t, i}^{a^{L}}
$$

These two modes of attack are equivalent as they both affect a customer's load response as long as part of the load is under cyber DSM control that is sensitive to price changes.

Theorem 1. Given a set of customers $\mathcal{A}$ compromised by the attacker, there always exists a direct load manipulation attack such that all customers behave the same as a pricing data injection attack and vice versa for $\kappa_{i}>0, \forall_{i}, t \in T_{a}$. 
Proof. Setting both attacks to have the same load, then the attack load is set as follows:

$$
a_{t, i}^{L}=\left(\kappa_{i} \phi_{t, i}\right)\left(a_{t, i}^{P}\right)^{\epsilon^{d s m}}+\left(1-\kappa_{i}\right) \phi_{t, i}
$$

and the equivalent attacked price is

$$
a_{t, i}^{P}=\left(\frac{a_{t, i}^{L}-\left(1-\kappa_{i}\right) \phi_{t, i}}{\kappa_{i} \phi_{t, i}}\right)^{1 / \epsilon^{d s m}}
$$

If $\kappa_{i}=0$, then the two attack modes are not equivalent since a price attack will have no effect on customer load.

Since false pricing data injection and direct load manipulation attacks are equivalent, we focus only on direct load manipulation attack analysis.

There are different goals an attacker can have to harm the power grid or exploit it. For example, an attacker can cause chaotic metering by messing the metering data transmission, efficiency loss of the energy provided by causing higher load volatility, or the energy system failure by overloading the power lines or devices. The focus of this work is efficiency loss by increasing user loads through direct load manipulation. In this scenario, we introduce three possible types of load attacks: ramp, sudden, and point attacks. These types of attacks are visualized in Figure 8 wherein plot (a) an attacker gradually increases a user's load over time. In plot (b), an attacker suddenly ramps up the power usage to a specified level, and, in plot (c), we demonstrate a point attack where the attacker increases loads only for specific hours.
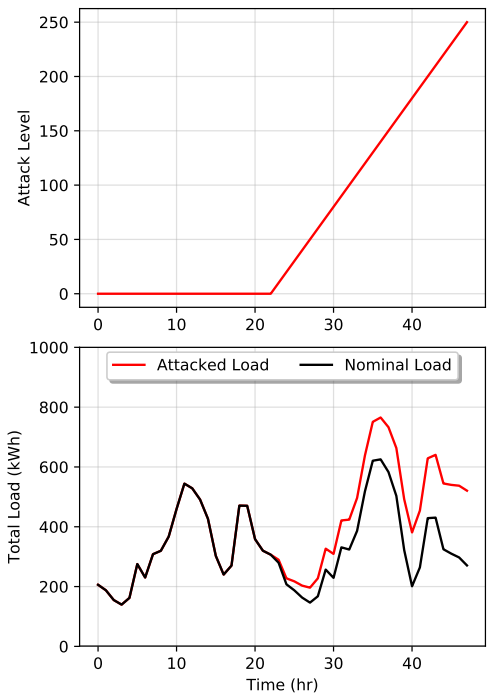

(a)
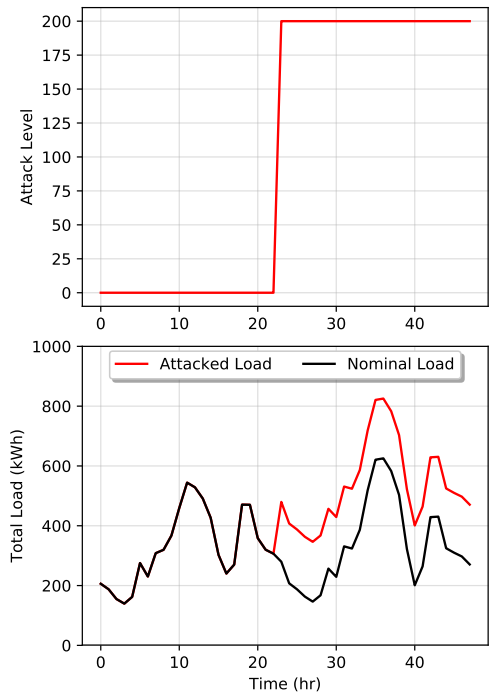

(b)
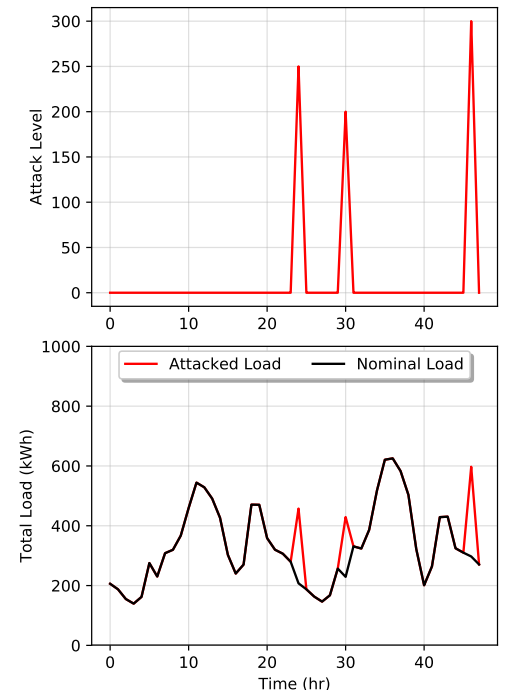

(c)

Figure 8. Examples of different type of DSM attacks: (a) ramp attack, (b) sudden attack, and (c) point attack.

\section{Attack Detection}

Here, we outline sequential parametric, supervised learning-based, and nonparametric methods for attack detection. The sequential detections methods are the one-sided CUmulative SUM (CUSUM) test [30] and windowed GLRT [31]. Both these methods take as an input a residual time series that is the output of applying a SARIMA filter to load observations. If enough past observations of load data labeled as nominal or under attack are collected, then detection of attacks can be made by training supervised learning classifiers. Supervised learning algorithms have been broadly adopted to the 
smart grid literature to monitor and detect cyber attacks on power systems [32-34]. Here, we employ several supervised learning methods for detecting attacks on cyber-DSM systems. It is unlikely that an ISO can collect high-quality attack data due to the lack of such attacks occurring. However, such data can be simulated. Using past load data, we simulate direct load manipulation attacks by creating different types of attacks, as shown in Figure 8.

\subsection{SARIMA Model}

In almost all our detection models, a Seasonal AutoRegressive Integrated Moving Average (SARIMA) forecast of load demand is used. Mathematically, the SARIMA model is denoted as $S A R I M A(p, d, q)(P, D, Q)(S)$, where $S$ refers to the number of time units in each season, $p$ and $q$ are the autoregressive and moving average terms for nonseasonal part, $d$ is the degree of differencing (the number of times the data have had past values subtracted), and the uppercase $P, D$, and $Q$ refer to the autoregressive, differencing, and moving average terms for the seasonal part of the ARIMA model. We can write the model as the following equation:

$$
\begin{aligned}
& \left(1-\sum_{i=1}^{p} \alpha_{i} B\right)\left(1-\sum_{i=1}^{P} \beta_{i} B^{S}\right)(1-B)^{d}\left(1-B^{S}\right)^{D} Y(t+1) \\
& =\left(1+\sum_{i=1}^{q} u_{i} B\right)\left(1+\sum_{i=1}^{Q} \gamma_{i} B^{S}\right) x(t+1)
\end{aligned}
$$

where $B$ is the back-shift operator of the model, $\alpha_{i}, \beta_{i}, u_{i}$, and $\gamma_{i}$ are fixed coefficients determined by the model after capturing the behavior of the series. $Y(t+1)$ is the price or load data at time $t+1$ and $x(t+1)$ is the residual of the data. The exponents in the equation represent the times that the back-shift operator is multiplied.

\subsection{Sequential Detection Methods}

In sequential change-point detection, a series does not have a fixed length. Instead, observations are received and processed sequentially over time. When an observation is received, a decision is made about whether a change has occurred in the state. This is based only on the observations that have been received so far or within a fixed past window size. If no change is detected, then the next observation in the sequence is processed. The sequential formulation allows sequences containing multiple change points to be easily handled. Sequential change-point detection can be applied in the case of attack detection to identify if a load time series has been compromised. If an attack is flagged, an ISO can take appropriate actions to prevent further damage to the grid.

In the parametric detection realm, the Generalized Likelihood Ratio Test (GLRT) is a popular and influential method; it has been widely used in anomaly detection and signal detection. The advantage of the GLRT is that it can provide estimates of the unknown shift in statistical parameters and has consequently been used to distinguish different types of anomalies [35]. However, its applicability relies on knowledge of the statistical parameters when a system is not under attack, and the effectiveness reduces with an increasing number of unknown parameters. In [36], H.B. Mann and D.R. Whitney first presented the theory of creating a new statistic for a sequence of data to detect a probabilistic change without knowing any distribution information of the data. In [37], Ming Yu illustrated several features for nonparametric detection in network anomaly detection: (1) no assumption is made on the distribution of observations, (2) its detection threshold is self-adjusted, and (3) it can react to the end of an anomaly within a required delay time. In [38], Hawkins employed an idea of dividing data into windows to analyze data anomaly in a window with the adjustable window size using a nonparametric method. In this work, we adapted Hawkins's idea and designed a moving window nonparametric detector to detect false data injection attacks in real time.

Under the Sequential Detection paradigm, we collect observations and apply a whitening filter to produce a continuing series with the assumption that it is white Gaussian noise. If an additive attack 
$A_{t}>0$ is present for observation $t$, this will cause a definite shift in the mean of the residual series. This detection problem can thus be stated as deciding if a null hypothesis $\mathcal{H}_{0}$ is true, where the residual series has zero mean and known variance (invariant in time and estimated from a sample population), or if the alternative hypothesis $\mathcal{H}_{1}$ is correct which states that the examined series has some mean not equal to zero, thus being under attack. This can be modeled as a hypothesis test, and, for the GLRT and CUSUM detectors, this translates to

$$
\begin{aligned}
& \mathcal{H}_{0}: x_{t} \sim \mathcal{N}\left(0, \sigma^{2}\right), t=1, \ldots, N \\
& \mathcal{H}_{1}: x_{t} \sim \mathcal{N}\left(A_{t}, \sigma^{2}\right), A_{t}>0, t=1, \ldots, N
\end{aligned}
$$

For the GLRT detector, to simplify implementation, we model the attack as if it were constant $A>0$ but unknown. To produce a residual series, a SARIMA multi-step forecast for $t=1, \ldots, N$ is made before the detection period. This forecast is conducted using a past window of training data that was not under attack. The forecast is made for time $t$ to $t+k$. These predictions are then subtracted from the incoming observations to produce a residual time series, which is then fed as input into the GLRT and CUSUM detectors.

\subsection{Nonparametric Detection Methods}

We look at two nonparametric detectors: CUSUM and Mann-Whitney. CUSUM is a classic nonparametric detection method. A CUSUM test is a control chart that is used to monitor the mean of a process based on samples taken from past data at specific time intervals. It is a class of nonlinear stopping rules for structural changes. Given information on current and previous samples, a CUSUM test relies on the specification of a target value $h$ and a known or reliable estimate of the standard deviation $\sigma$ the process. The CUSUM test typically signals an out of control or anomalous process by an upward or downward drift of the cumulative sum until it crosses the target threshold. If the mean of the load series shifts above the target threshold for attack detection, we assume the grid is under attack.

We define the CUSUM detector as follows. Taking the residual series $x_{t}=y_{t}-E_{t-1}\left[y_{t}\right]$, again defined by a SARIMA forecast $E_{t-1}\left[y_{t}\right]$, we define a one-sided CUSUM detector as

$$
g_{t}=\max \left(0, g_{t-1}+x_{t}-k\right)
$$

where $k$ is called the reference value (sometimes also called drift) set priori to values such as $0,0.5$, or $A / 2$ if the size of $A$ is known in advance. When $g_{t}=0$, then we define the change time as $t_{c}=t$, and, when $g_{t}>h>0$, we reset $g_{t}=0$ and flag an alarm at time $t_{a}=T$. The alarm threshold is also set priori to some value based on the sample population standard deviation such as $h=2 \sigma$, where $\sigma$ is estimated from past training data used to produce the SARIMA forecasts.

Building off the CUSUM, a more sophisticated nonparametric detector is the Mann-Whitney method. Following the approach in [36], we consider a sequence of data samples sorted into descending order. Each of them has $k$ elements from set $X$ and $n-k$ elements from set $Y$. We label elements from $Y$ as $a_{1}, X$ as $a_{0}$. Let $P_{k, n-k}(U)$ represent the probability of a sequence in which $a_{1}$ precedes $a_{0} U$ times. We define the following statistic from the data

$$
U_{k, n-k}=\sum_{i=k+1}^{n} \sum_{j=1}^{k} \psi\left(X_{i}-X_{j}\right)
$$

where $\psi(t)$ is the unit step function $(\psi(t)=0, t<0$ and $\psi(t)=1, t \geq 0)$. Consequently, we can write

$$
P_{k, n-k}(U)=\frac{k}{n} P_{k-1, n-k}(U-n+k)+\frac{n-k}{n} P_{k, n-k-1}(U)
$$


Following the approach in [36], we get

$$
\begin{array}{r}
E_{k, n-k}(U)=\frac{k(n-k)}{2} \\
\operatorname{Var}_{k, n-k}(U)=\frac{k(n-k)(n+1)}{12}
\end{array}
$$

Placing the $n$ data points into a window and utilizing $k$ as a moving split point, the pre split sequence is $a_{0}$ and the post-split sequence is $a_{1}$.

When the data are under attack, by computing $U_{k, n-k}$ for different values of $k$, the detector can determine a suspect start point of the attack. The algorithm can be illustrated by the following equation:

$$
\begin{aligned}
T_{\text {max }, k, n-k} & =\max _{1 \leq k \leq n-1}\left\{\frac{\left|U_{k, n-k}-\frac{k(n-k)}{2}\right|}{\sqrt{\frac{k(n-k)(n+1)}{12}}}\right\} \\
\overline{\mathcal{T}}_{T} & =\arg \max _{1 \leq k \leq n-1}\left(T_{\text {max }, k, n-k}\right)
\end{aligned}
$$

where $T_{k, n-k}$ is the test statistic of the detector, $T_{\max , k, n-k}$ is the maximum value of the $T_{k, n-k}$ by traversing $k$ in $1 \leq k \leq n-1$ and $\overline{\mathcal{T}}_{T}$ is the corresponding $k$ when the $T_{\max , k, n-k}$ is located. With all previous discussion, we can introduce a specified control limit $h_{n}$ and create a hypothesis test:

- Compute $T_{\max , k, n-k}$, the maximized split statistic.

- If $T_{\max , k, n-k} \leq h_{n}$, then conclude that the data in this window is in control without attack and continue to move the window forward along the whole sequence of data.

- If $T_{\max , k, n-k}>h_{n}$, then conclude that the data in this window is under attack and stop the process of moving the window for diagnosis. Estimate the epoch of attack start point by the maximizing $k$, which is $\overline{\mathcal{T}}_{T}$.

\subsection{Supervised Learning Methods}

Changepoint detection could alternatively be seen as a supervised learning binary classification problem. Under this scheme, all of the change point sequences, or in our case attacks, represent one class, and all of the nominal sequences represent a second class. Supervised learning methods are machine learning algorithms that learn a mapping from input data to a target class label. Given a set of samples $\mathcal{X}=\left\{x_{i}\right\}_{i=1}^{N}$ and a set of labels $\mathcal{Y}=\left\{y_{i}\right\}_{i=1}^{N}$, then the supervised learning detection problem is defined as a hypothesis function that captures the relationship between samples and labels $f: \mathcal{X} \longrightarrow \mathcal{Y}$. A sliding window moves through the data, considering each difference between two data points as a possible change point.

An advantage of treating attack detection as a supervised learning problem is that it has a more straightforward training phase. However, a sufficient amount and diversity of training data need to be provided to represent all of the classes of attacks. To ensure enough training data, and to prevent class label imbalance, we simulate all attack data to train our algorithms. Machine learning methods have successfully been applied several times in data injection attacks in power systems [33,34], so we analyze here their ability to detect data attacks on cyber-DSM systems. The binary classification problem for attack detection can be defined as

$$
y_{i}=\left\{\begin{array}{l}
1, \text { if } A_{i}>0 \\
0, \text { if } A_{i}=0
\end{array}\right.
$$

where $y_{i}=1$ if the $i$-th observation is under attack, and $y_{i}=0$ if there is no attack. A variety of classifiers can be used for this learning problem. For detection of DSM attacks, we examine Logistic Regression (LR), Random Forests (RF), Gaussian Naive Bayes (GNB), Gradient Boosting Classifier (GBC), and an Artificial Neural Network (ANN). We chose these classifiers because they are all compelling and have widespread use in both industry and academia; model descriptions of these 
methods can be found in [39,40]. All these models were implemented using the Python package scikit-learn [41] with each model using default parameters from the package.

\subsection{Performance Analysis}

For the security of cyber DSM systems, the major concern is not just the detection of attacks, but also the detection of nominal data with high reliability. We want a detection system that can predict not only with high accuracy but also with high precision and recall to avoid false alarms. Therefore, we measure the True Positives (TP), the True Negatives (TN), the False Positives (FP), and the False Negatives (FN). Definitions of these measures are visually shown in Figure 9. We use these measures to calculate several main performance indicators of accuracy, precision, and recall.

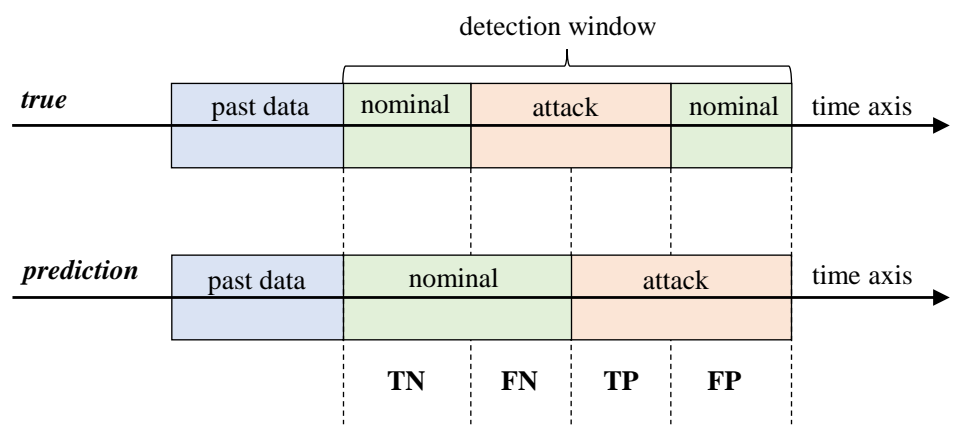

Figure 9. Confusion matrix imposed on a time axis of attack predictions vs. true observations.

We calculate accuracy as the ratio of correctly classified data points to total data points

$$
\text { Accuracy }=\frac{T P+T N}{T P+T N+F P+F N}
$$

This measure provides the total classification success of the models. However, accuracy is not enough to get a full picture of performance alone. Precision is calculated as the ratio of true positive data points (attacks) to total points classified as attacks:

$$
\text { Precision }=\frac{T P}{T P+F P}
$$

On the other hand, recall, also known as the True Positive Rate (TPR), refers to the portion of attacks that were recognized correctly

$$
\text { Recall }=\frac{T P}{T P+F N}
$$

Precision values give information about the prediction performance of the algorithms, whereas recall values measure the degree of attack retrieval. For instance, a recall value equal to 1 signifies that none of the attacked measurements were misclassified as nominal.

We use one more final measure of total performance of our detectors, the Receiver Operating Characteristics (ROC) curve. The ROC curve is an assessment that enables visual analysis of the trade-off between TPR and False Positive Rate (FPR). This can also be seen as the trade-off between the probability of detection and the probability of false alarm. FPR is defined as follows:

$$
F P R=\frac{F P}{F P+T N}
$$


The ROC curve is constructed by plotting a two-dimensional graph with false positive rate on the $x$-axis and true positive rate on the $y$-axis at various threshold settings. A detection algorithm produces a (TPR, FPR) pair that corresponds to a single point in the ROC space. An ROC curve that's close to the upper axes boundary is reflective of a good detector. The best possible detection method would produce a point in the upper left corner, coordinate $(0,1)$ of the ROC space. A random prediction would give a point along a diagonal line from $(0,0)$ to $(1,1)$. Points above this line are considered to have performance, while points below are considered with performance worse than guessing.

\section{Detection Experiments and Results}

For attack simulation and detection experiments, we use all the same parameters from Table 1 , but we varied the levels of $\kappa$ and attacks $a_{t}$. We simulate each of the three types of attacks, as visualized in Figure 8, each in the form of direct load manipulation attack, under DSM participation levels $\kappa=0.1$ and 0.9. This creates a total of six experiment scenarios. For each scenario, we simulate 28 days of training data (672 observations) and two proceeding days of test data (48 observations), both at a resolution of $1 \mathrm{hr}$. All training data are created nominally with no attacks. In the test data sets, the first $24 \mathrm{hrs}$ of the test data are not under attack. In the last $24 \mathrm{~h}$ of the test data, we add one of the three types of attacks. For ramp attacks, for each time step, we add an attack $a_{t}=a_{t-1}+5$ where the first $24 \mathrm{~h}$ of the test set $a_{t=0 . .23}=0$. The sudden attacks are similar except the attack is an additive constant $a_{t}=150$. The last type of attack, point attacks, are $a_{t=24}=250, a_{t=29}=200, a_{t=34}=300, a_{t=37}=100, a_{t=46}=150$, and $a_{t}=0$ everywhere else. To showcase a wide variety of detection strategies, we conduct two main studies. In the first, we compare sequential detectors such as GLRT and CUSUM to five machine learning-based classifiers using accuracy, recall, precision, and ROC curves. In the second study, we do a deep analysis comparing GLRT to nonparametric based detection with ROC curves.

\subsection{Sequential vs Supervised Learning Detection}

For the sequential detectors, we use multi-step SARIMA forecasts to predict the next $48 \mathrm{~h}$ and then use those forecasts to filter incoming test data for detection. The training was conducted on the training time series of 28 days of nominal data. SARIMA hyperparameters were chosen by examining lag one differenced AutoCorrelation Function (ACF) and Partial AutoCorrelation Function (PACF) plots of the training data. These forecasts where then subtracted from the incoming test data to obtain a residual series that is input to the sequential detectors. The assumption for both detectors is that the residual series is Gaussian white noise, where the series has zero mean, and each observation is independent identically distributed from a Gaussian distribution.

To ensure the residuals are white noise, we apply the Augmented Dickey-Fuller (ADF) test to check for stationarity, and we examine the ACF/PACF plots to check for independence and run a Jarque-Bera test, and we examine a Q-Q plot to check if the residual series has a Gaussian probability distribution. We conduct these checks on all scenario training datasets, where we first train a SARIMA fit on them and then subtract that fit to produce the residual series. In all the training data sets, the residuals were stationary from the ADF test and independent from ACF/PACF plots. However, the Jarque-Bera test and Q-Q plots showed that the observations did not come from a Gaussian distribution. Example, ACF/PACF, and Q-Q plots for $\kappa=0.1$ are shown in Figures 10 and 11. Despite the residuals not being Gaussian, we still run the sequential detectors and examine their performance. 

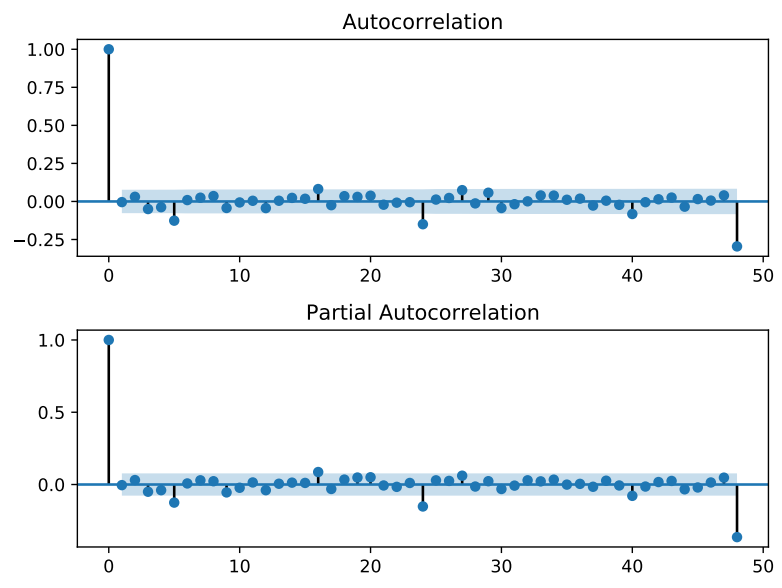

Figure 10. ACF and PACF plots of the residual series between the SARIMA fit tp training data. From the plots, we observe that the residuals are stationary.

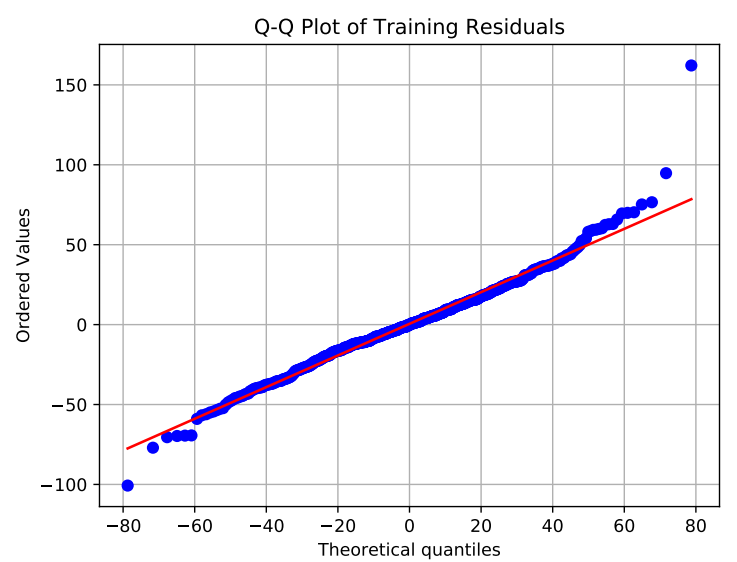

Figure 11. $Q-Q$ plot of the residual series between the SARIMA fit tp training data. From the plot, we observe that, for extreme quantiles, the distribution is not Gaussian.

For training, the supervised learning methods for each of the six scenarios, we simulate more data to ensure proper class learning. We keep the test sets the same, but we extend each training set doubling its size. We keep the original training set, labeling it as nominal, and then make a copy of it. In the copy, we split it into three parts; for each part, we add one of the types of attacks at random levels. We label each observation of this set as under attack. We added the nominal and attacked training sets to form a new training set with a total of 1344 observations. With our training and test time series, we then create a set $\mathcal{X}$ features and $\mathcal{Y}$ labels that could be fed into the supervised learning classifiers. Each training sample $x_{i} \in \mathcal{X}$ is composed of $24 \mathrm{~h}$ of lagged data, each hour is one feature, and each label $y_{i} \in \mathcal{Y}$ corresponds to a class 0 (not under attack) and class 1 (under attack). Together, we get $N=1298$ pairs of $\left(x_{i}, y_{i}\right)$ training samples.

We run our experiments on a computer with an Intel i7 $67002.6 \mathrm{GHz}$ and $16 \mathrm{~GB}$ of RAM. Implementation of the simulations, experiments, and sequential detectors were done in Python 3.6. Implementation for SARIMA forecasts was done using the Python package Statsmodels [42], the supervised learning methods, and confusion evaluation metrics were implemented using the Scikit-Learn Python package [41], and ROC curves were created using the Matplotlib Python package [43]. All classifiers used default hyperparameters from Scikit-Learn. We compiled all code and data used in our work into a Python package titled LehighDSM, which is publicly available on GitHub [44]. 
Experimental results from the six scenarios are reported in Table 2, in the form of accuracy, recall, and precision metrics, and Figure 12 which showcases six ROC curves, one for each scenario. All performance measures in Table 2 were calculated using the best thresholds found in the ROC curves by searching for the shortest distance from each curve to the corner $(0,1)$. As seen in Figure $12 \mathrm{c}, \mathrm{f}$ type 3 attacks, under any DSM level, had the lowest detection rates across thresholds with ANN being the worst performer. In Figure 12a,d, LR yielded the best performance with GNB, and again ANN being the worst. Type 2 attacks in Figure 12b,e resulted in the best detection with CUSUM, LR, GNB, and GBC having perfect performance.

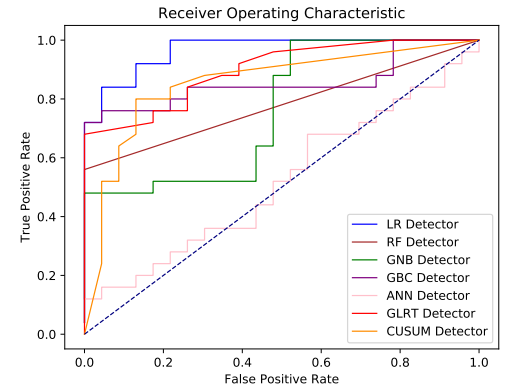

(a) Type 1 attack; $\kappa=0.1$

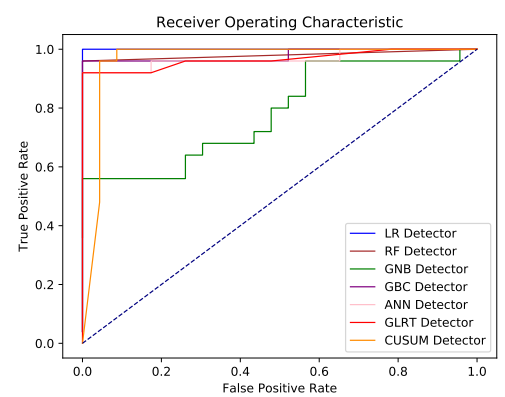

(d) Type 1 attack; $\kappa=0.9$.

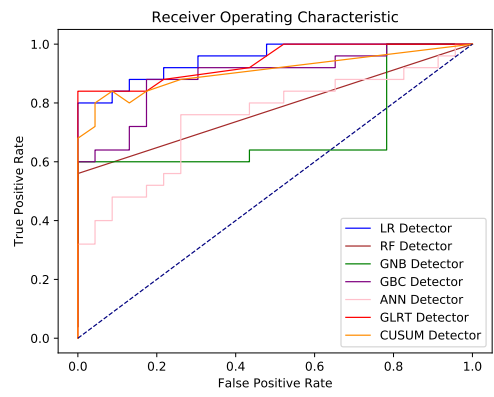

(b) Type 2 attack; $\kappa=0.1$

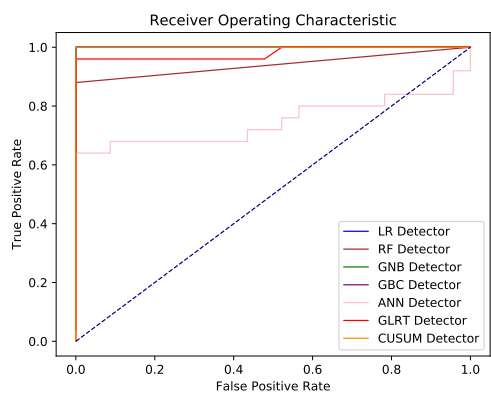

(e) Type 2 attack; $\kappa=0.9$.

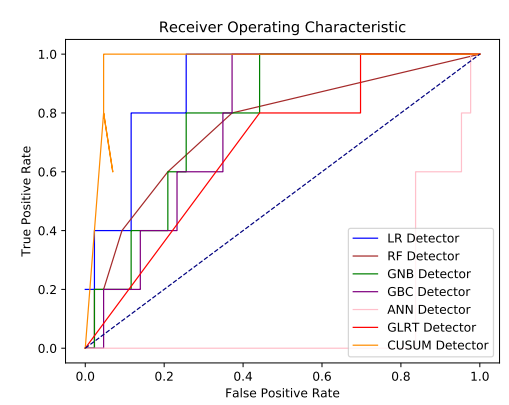

(c) Type 3 attack; $\kappa=0.1$.

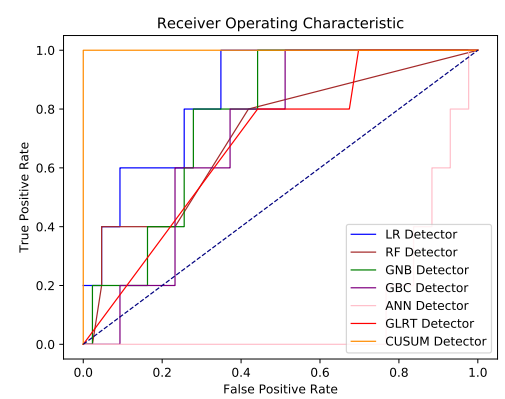

(f) Type 3 attack; $\kappa=0.9$

Figure 12. ROC curves for the six different experiment scenarios.

With the results from Table 2, we have the following conclusions: demonstrated findings imply that detection of attacks has a higher accuracy with higher levels of DSM participation. This occurs since higher DSM penetration results in more aggregated attacks. However, we note that an attacker, if they have perfect knowledge of the grid, could decrease their intensity and make detection more difficult. Furthermore, the supervised learning classifiers' performance on average was on par or better than sequential detection methods. From a detector comparison perspective, the parametric and nonparametric detectors showcased higher performance on most of the attacks and varying parameters. In the following section, we do a deeper dive on a comparison between the GLRT and the nonparametric detectors to derive further inferences on operations paradigms for the attack detection problem. 
Table 2. Evaluation metrics for attack detection. Best results in bold.

\begin{tabular}{|c|c|c|c|c|c|c|c|}
\hline & $\kappa$ & & 0.1 & & & 0.9 & \\
\hline & Attack Type & 1 & 2 & 3 & 1 & 2 & 3 \\
\hline \multirow{7}{*}{ 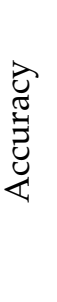 } & LR & 89.6 & 100.0 & 87.5 & 87.5 & 100.0 & 75.0 \\
\hline & RF & 77.1 & 97.9 & 64.6 & 77.1 & 93.8 & 60.4 \\
\hline & GNB & 70.8 & 77.1 & 75.0 & 79.2 & 100.0 & 72.9 \\
\hline & GBC & 85.4 & 97.9 & 66.7 & 85.4 & 100.0 & 64.6 \\
\hline & ANN & 56.3 & 95.8 & 20.8 & 75.0 & 79.2 & 14.6 \\
\hline & GLRT & 79.2 & 95.8 & 58.3 & 91.7 & 97.9 & 58.3 \\
\hline & CUSUM & 83.3 & 95.8 & 95.8 & 87.5 & 100.0 & 100.0 \\
\hline \multirow{7}{*}{$\begin{array}{l}\overline{\widetilde{U}} \\
\stackrel{\mathscr{U}}{\simeq}\end{array}$} & LR & 92.0 & 100.0 & 80.0 & 89.0 & 100.0 & 80.0 \\
\hline & RF & 56.0 & 96.0 & 80.0 & 56.0 & 88.0 & 80.0 \\
\hline & GNB & 88.0 & 56.0 & 80.0 & 60.0 & 100.0 & 80.0 \\
\hline & GBC & 76.0 & 96.0 & 100.0 & 88.0 & 100.0 & 80.0 \\
\hline & ANN & 68.0 & 92.0 & 60.0 & 76.0 & 68.0 & 80.0 \\
\hline & GLRT & 76.0 & 92.0 & 80.0 & 84.0 & 96.0 & 80.0 \\
\hline & CUSUM & 80.0 & 96.0 & 100.0 & 84.0 & 100.0 & 100.0 \\
\hline \multirow{7}{*}{ 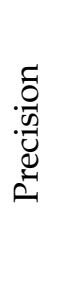 } & LR & 88.5 & 100.0 & 44.4 & 88.0 & 100.0 & 26.7 \\
\hline & RF & 100.0 & 100.0 & 20.0 & 100.0 & 100.0 & 18.2 \\
\hline & GNB & 66.7 & 100.0 & 26.7 & 100.0 & 100.0 & 25.0 \\
\hline & GBC & 95.0 & 100.0 & 23.8 & 84.0 & 100.0 & 20.0 \\
\hline & ANN & 56.7 & 100.0 & 7.7 & 76.0 & 89.5 & 9.1 \\
\hline & GLRT & 82.6 & 100.0 & 17.4 & 100.0 & 100.0 & 17.4 \\
\hline & CUSUM & 87.0 & 96.0 & 71.4 & 91.3 & 100.0 & 100.0 \\
\hline
\end{tabular}

\subsection{GLRT Detection versus Nonparametric Detection}

For the comparison between the nonparametric detector and parametric GLRT detector, we conduct our numerical experiments using the block bootstrap approach on real demand data (unmodulated by DSM) available from the New England ISO, to generate demand for 7 to 200 users. We apply the price-elasticity model with the DSM participating rate of $k$ and pricing information from the New England ISO to generate the DSM modulated price and demand for multiple time periods. We first compare the performance of the GLR and nonparametric detectors on identical data windows in Figures 13 and 14. $P$ denotes the DSM participation rate, $k$ means the percentage of the data in the selected window is under attack. Using twin attacks, we compare the detectors using ROC curves.

There are a few key observations from Figures 13 and 14. First, as expected, the performance of both detectors improves with attack magnitude. Second, the nonparametric detector outperforms the GLRT for small user sizes, whereas the comparison yields the opposite conclusion in the 200 user simulations. The reasoning behind this observation is that: when fewer users' data are analyzed, the residuals based on the SARIMA forecasts are far less likely to behave like white noise, and, consequently, the GLRT optimized for Gaussian residuals underperforms. The nonparametric detector, aside from not requiring the legitimate statistics, purely checks for statistical shifts and performs better in a smaller user pool. In a larger user pool, we enter a law of large numbers regime, where the aggregate residuals increasingly resemble Gaussian variables, and thus the optimized GLRT has a better ROC curve.

Figure 15 plots the performance comparison as a function of attack magnitude and window sizes for a fixed false alarm probability $\left(P_{F A}\right)$. As expected, the GLRT outperforms the nonparametric detector for larger window sizes regardless of attack magnitude. With smaller window sizes and, consequently, shorter delay in detection, the nonparametric detector outperforms the GLRT, which illustrates that, when statistical variations are small (due to fewer users, smaller attack magnitude or lower delay), nonparametric detectors are appropriate. 


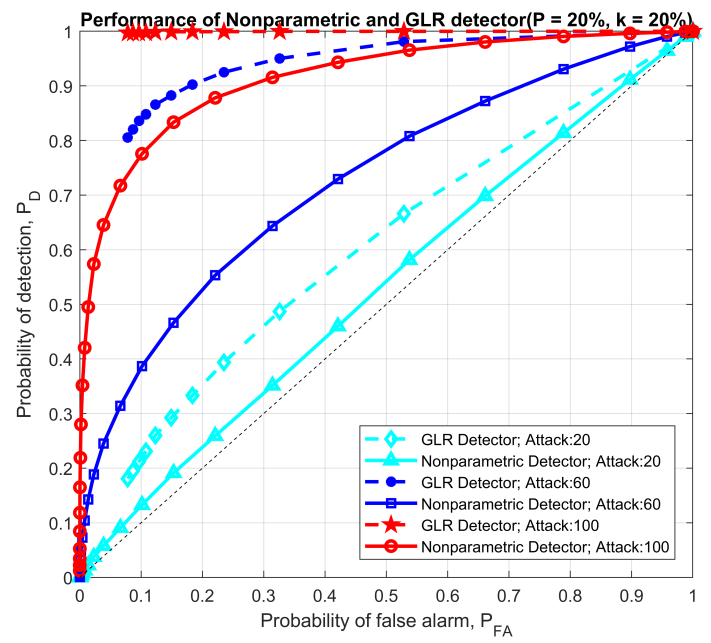

Figure 13. Performance comparison between parametric and nonparametric detector under 200 users.

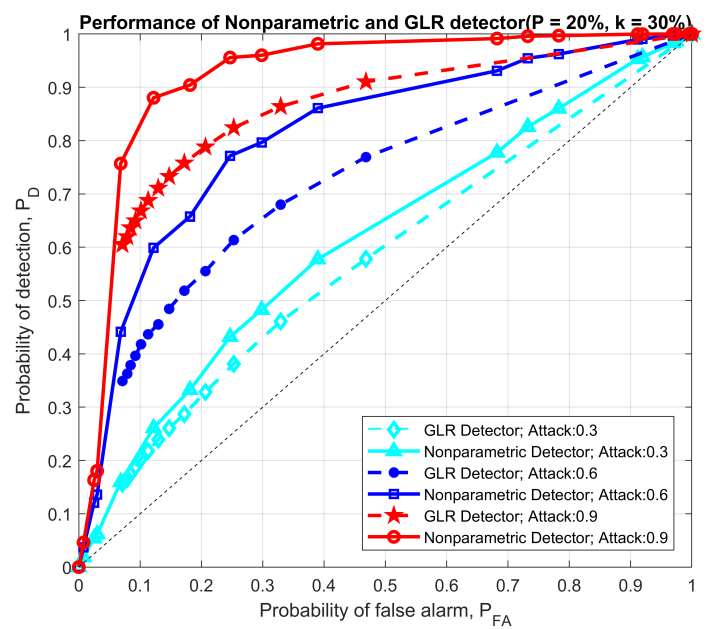

Figure 14. Performance comparison between parametric and nonparametric detectors with two users only.

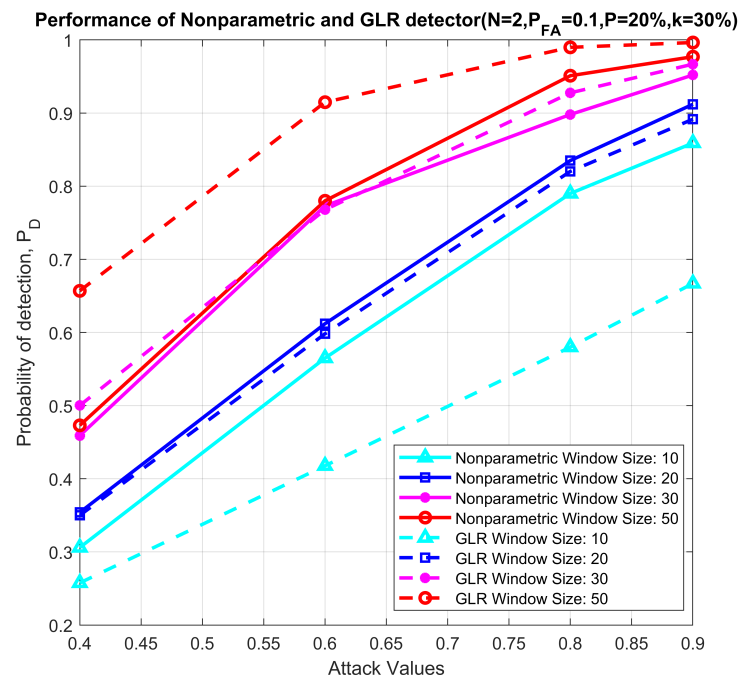

Figure 15. Performance comparison between nonparametric and GLRT detector, $\mathrm{N}=2, P_{F A}=0.1$, $P=20 \%, \mathrm{k}=30 \%$. 


\section{Conclusions}

In this work, we study the exploitation of the hypothetical feedback between cyber-enabled DSM programs, on the consumer side, and dynamic RTP on the utility side. An attacker with exploitive economic or nefarious intentions, such as causing efficiency loss of energy provision, can take advantage of the dependency between dynamic pricing and DSM load control. The utility modifies prices in response to forecasted demand in order to push realized load up or down. This is done to achieve some target load level to reduce peak load or achieve some other DSM objective. On the user side, cyber DSM programs then autonomously respond to prices to adjust certain portions of a user's load up or down with some given elasticity.

We propose two modes of attacks, false price data injections, and direct load manipulation. Under a false price data injection, an attacker modifies the RTP that users receive to alter their demand. Through a direct load manipulation attack, attacker hacks and alters a user's load profile directly. In both of these attacks, aggregate load from the grid is modified, which can alter future prices or demand. We showcase how these two modes of attacks are equivalent and introduce three ways an attack can occur. The first type is a ramp attack, the second is a sudden attack, and the third is a point attack. We simulate these types of attacks and review several methods to detect them.

We simulate and examine load-price data under different DSM participation levels with three types of additive random attacks: ramp, sudden, and point attacks. We then conduct two tutorial-style detection studies. First, we compare sequential change point and supervised learning methods. Moreover, in a second study, we compare parametric and nonparametric detectors under different conditions. These two studies are meant to provide a broad review of various possible detection approaches. The proposed approaches are pure data driven approaches which can be implemented without additional hardware assuming that the data are collected at a demand response management system. The computational cost of the proposed detection methods would depend on the specific methodologies adopted. Statistical tests can be implemented without incurring significant computational cost whereas more complex detectors such as ANNs require hardware acceleration for reasonable performance.

Results conclude that higher amounts of DSM participation can exacerbate attacks and lead to better detection of such attacks, point attacks are the hardest to detect, and supervised learning methods produce par or better results than sequential detectors. Amongst the GLRT based parametric and the nonparametric detector, we show that, with growing window size, the performance of both the detectors will increase. The performance of the GLRT detector increases faster when the detection horizon increases due to the MLE mechanism. Thus, if the detection horizon is broad and the user number is fixed, both the detectors become more potent due to the more information and larger size of the attack data.

Due to the speculative nature of these types of attacks, we plan to look into many different issues for future studies. All our detection approaches for attacks depend on predictions. When an accurate detection is made, the ISO would take measures to mitigate that attack by adjusting price levels appropriately. In the event of false negatives or false positives, for part of the future work, we envision to study how an ISO could have a risk cost matrix that would define the amount of price adjustments for which it would feel comfortable to mitigate attacks taking into account probability confidence levels on the accuracy of the attacks. Additional new directions include expanding our model to incorporate day ahead pricing and DSM scheduling, adding models of cost and utility on for a customer and ISO, expanding our model taking into account grid topology, and modeling more advanced attack goals such as system failure by power line overload. Additionally, in this work, we only looked at the DSM goal of strategic load conservation. More complex strategies, such as load shifting, could result in more complex types of cyber attacks; this also makes for compelling future work. 
Author Contributions: Conceptualization, methodology, investigation, software K.H.; nonparametric detection investigation, C.Z.; writing-original draft preparation, K.H. and C.Z.; writing-review and editing, P.V., S.K., and R.S.B.; supervision, P.V. and S.K.; funding acquisition, P.V., S.K., R.S.B., and L.S. All authors have read and agreed to the published version of the manuscript.

Funding: This work is supported by the Department of Energy through Award Number DE-OE0000779.

Acknowledgments: The authors thank the anonymous reviewers for their careful reading of our manuscript and their many insightful comments and suggestions.

Conflicts of Interest: The authors declare no conflict of interest.

\section{References}

1. Faruqui, A.; Sergici, S. Household Response to Dynamic Pricing of Electricity-a Survey of The Empirical Evidence. 2010. Available online: https://papers.ssrn.com/sol3/papers.cfm?abstract_id=1134132 (accessed on 5 April 2019).

2. Grochocki, D.; Huh, J.H.; Berthier, R.; Bobba, R.; Sanders, W.H.; Cárdenas, A.A.; Jetcheva, J.G. AMI threats, intrusion detection requirements and deployment recommendations. In Proceedings of the 2012 IEEE Third International Conference on Smart Grid Communications (SmartGridComm), Tainan, Taiwan, 5-8 November 2012; pp. 395-400.

3. Khan, A.R.; Mahmood, A.; Safdar, A.; Khan, Z.A.; Khan, N.A. Load forecasting, dynamic pricing and DSM in smart grid: A review. Renew. Sustain. Energy Rev. 2016, 54, 1311-1322. [CrossRef]

4. Xie, L.; Mo, Y.; Sinopoli, B. Integrity data attacks in power market operations. IEEE Trans. Smart Grid 2011, 2, 659-666. [CrossRef]

5. Tan, R.; Badrinath Krishna, V.; Yau, D.K.; Kalbarczyk, Z. Impact of integrity attacks on real-time pricing in smart grids. In Proceedings of the 2013 ACM SIGSAC Conference on Computer \& Communications Security, Berlin, Germany, 4-8 November 2013; ACM: New York, NY, USA, 2013; pp. 439-450.

6. Palensky, P.; Dietrich, D. Demand side management: Demand response, intelligent energy systems, and smart loads. IEEE Trans. Ind. Inform. 2011, 7, 381-388. [CrossRef]

7. Logenthiran, T.; Srinivasan, D.; Shun, T.Z. Demand side management in smart grid using heuristic optimization. IEEE Trans. Smart Grid 2012, 3, 1244-1252. [CrossRef]

8. Mohsenian-Rad, A.H.; Wong, V.W.; Jatskevich, J.; Schober, R.; Leon-Garcia, A. Autonomous demand-side management based on game-theoretic energy consumption scheduling for the future smart grid. IEEE Trans. Smart Grid 2010, 1, 320-331. [CrossRef]

9. Gelazanskas, L.; Gamage, K.A. Demand side management in smart grid: A review and proposals for future direction. Sustain. Cities Soc. 2014, 11, 22-30. [CrossRef]

10. Batista, A.C.; Batista, L.S. Demand Side Management using a multi-criteria epsilon-constraint based exact approach. Expert Syst. Appl. 2018, 99, 180-192. [CrossRef]

11. Dudek, G. Pattern-based local linear regression models for short-term load forecasting. Electr. Power Syst. Res. 2016, 130, 139-147. [CrossRef]

12. Pappas, S.S.; Ekonomou, L.; Karampelas, P.; Karamousantas, D.; Katsikas, S.; Chatzarakis, G.; Skafidas, P. Electricity demand load forecasting of the Hellenic power system using an ARMA model. Electr. Power Syst. Res. 2010, 80, 256-264. [CrossRef]

13. Christiaanse, W. Short-term load forecasting using general exponential smoothing. IEEE Trans. Power Appar. Syst. 1971, PAS-90, 900-911. [CrossRef]

14. Taylor, J.W. Triple seasonal methods for short-term electricity demand forecasting. Eur. J. Oper. Res. 2010, 204, 139-152. [CrossRef]

15. Kavousi-Fard, A.; Samet, H.; Marzbani, F. A new hybrid modified firefly algorithm and support vector regression model for accurate short term load forecasting. Expert Syst. Appl. 2014, 41, 6047-6056. [CrossRef]

16. Baliyan, A.; Gaurav, K.; Mishra, S.K. A review of short term load forecasting using artificial neural network models. Procedia Comput. Sci. 2015, 48, 121-125. [CrossRef]

17. Kong, W.; Dong, Z.Y.; Jia, Y.; Hill, D.J.; Xu, Y.; Zhang, Y. Short-term residential load forecasting based on LSTM recurrent neural network. IEEE Trans. Smart Grid 2017, 10, 841-851. [CrossRef] 
18. Dudek, G. Short-term load forecasting using random forests. In Intelligent Systems'2014, Proceedings of the 7th IEEE International Conference Intelligent Systems IS'2014, Warsaw, Poland, 24-26 September 2014; Springer: Berlin/Heidelberg, Germany, 2015; pp. 821-828.

19. Li, S.; Goel, L.; Wang, P. An ensemble approach for short-term load forecasting by extreme learning machine. Appl. Energy 2016, 170, 22-29. [CrossRef]

20. Chakraborty, S.; Ito, T.; Senjyu, T. Smart pricing scheme: A multi-layered scoring rule application. Expert Syst. Appl. 2014, 41, 3726-3735. [CrossRef]

21. Nazar, N.; Abdullah, M.; Hassan, M.; Hussin, F. Time-based electricity pricing for Demand Response implementation in monopolized electricity market. In Proceedings of the 2012 IEEE Student Conference on Research and Development (SCOReD), Pulau Pinang, Malaysia, 5-6 December 2012; IEEE: Piscataway, NJ, USA, 2012; pp. 178-181.

22. Zimmerman, R.D.; Murillo-Sánchez, C.E.; Gan, D. MATPOWER: A MATLAB Power System Simulation Package; Manual, Power Systems Engineering Research Center: Ithaca, NY, USA, 1997; Volume 1.

23. Chassin, D.P.; Schneider, K.; Gerkensmeyer, C. GridLAB-D: An open-source power systems modeling and simulation environment. In Proceedings of the 2008 IEEE/PES Transmission and Distribution Conference and Exposition, Chicago, IL, USA, 21-24 April 2008; IEEE: Piscataway, NJ, USA, 2008; pp. 1-5.

24. Barker, S.; Mishra, A.; Irwin, D.; Cecchet, E.; Shenoy, P.; Albrecht, J. Smart*: An open data set and tools for enabling research in sustainable homes. Sustkdd August 2012, 111, 108.

25. Brockwell, P.J.; Davis, R.A.; Calder, M.V. Introduction to Time Series and Forecasting; Springer: Berlin/Heidelberg, Germany, 2002; Volume 2.

26. Gamerman, D.; Lopes, H.F. Markov Chain Monte Carlo: Stochastic Simulation for Bayesian Inference; Chapman and Hall/CRC: Boca Raton, FL, USA, 2006.

27. Politis, D.N.; White, H. Automatic block-length selection for the dependent bootstrap. Econom. Rev. 2004, 23, 53-70. [CrossRef]

28. Thimmapuram, P.R.; Kim, J.; Botterud, A.; Nam, Y. Modeling and simulation of price elasticity of demand using an agent-based model. In Proceedings of the 2010 Innovative Smart Grid Technologies (ISGT), Gothenburg, Sweden, 19-21 January 2010; IEEE: Piscataway, NJ, USA, 2010; pp. 1-8.

29. Liang, G.; Zhao, J.; Luo, F.; Weller, S.R.; Dong, Z.Y. A review of false data injection attacks against modern power systems. IEEE Trans. Smart Grid 2017, 8, 1630-1638. [CrossRef]

30. Tartakovsky, A.; Nikiforov, I.; Basseville, M. Sequential Analysis: Hypothesis Testing and Changepoint Detection; Chapman and Hall/CRC: Boca Raton, FL, USA, 2014.

31. Key, S. Fundamentals of Statistical Signal Processing; Volume ii: Detection Theory; Prentice Hall PTR: Upper Saddle River, NJ, USA, 1993.

32. Hink, R.C.B.; Beaver, J.M.; Buckner, M.A.; Morris, T.; Adhikari, U.; Pan, S. Machine learning for power system disturbance and cyber-attack discrimination. In Proceedings of the 20147 th International Symposium on Resilient Control Systems (ISRCS), Denver, CO, USA, 19-21 August 2014; pp. 1-8.

33. Ozay, M.; Esnaola, I.; Vural, F.T.Y.; Kulkarni, S.R.; Poor, H.V. Machine learning methods for attack detection in the smart grid. IEEE Trans. Neural Netw. Learn. Syst. 2016, 27, 1773-1786. [CrossRef]

34. Esmalifalak, M.; Liu, L.; Nguyen, N.; Zheng, R.; Han, Z. Detecting stealthy false data injection using machine learning in smart grid. IEEE Syst. J. 2017, 11, 1644-1652. [CrossRef]

35. Apley, D.W.; Shi, J. The GLRT for statistical process control of autocorrelated processes. IIE Trans. 1999, 31, 1123-1134. [CrossRef]

36. Mann, H.B.; Whitney, D.R. On a test of whether one of two random variables is stochastically larger than the other. Ann. Math. Stat. 1947, 18, 50-60. [CrossRef]

37. Yu, M. A nonparametric adaptive CUSUM method and its application in network anomaly detection. Int. J. Adv. Comput. Technol. 2012, 4, 280-288.

38. Hawkins, D.M.; Deng, Q. A nonparametric change-point control chart. J. Qual. Technol. 2010, 42, $165-173$. [CrossRef]

39. Bishop, C. Pattern Recognition and Machine Learning. In Pattern Recognition and Machine Learning; Springer: Berlin/Heidelberg, Germany, 2006.

40. James, G.; Witten, D.; Hastie, T.; Tibshirani, R. An Introduction to Statistical Learning; Springer: Berlin/Heidelberg, Germany, 2013; Volume 112. 
41. Pedregosa, F.; Varoquaux, G.; Gramfort, A.; Michel, V.; Thirion, B.; Grisel, O.; Blondel, M.; Prettenhofer, P.; Weiss, R.; Dubourg, V.; et al. Scikit-learn: Machine learning in Python. J. Mach. Learn. Res. 2011, 12, 2825-2830.

42. Seabold, S.; Perktold, J. Statsmodels: Econometric and statistical modeling with python. In Proceedings of the 9th Python in Science Conference, SciPy Society Austin, Austin, TX, USA, 28 June-3 July 2010; Volume 57, p. 61.

43. Hunter, J.D. Matplotlib: A 2D graphics environment. Comput. Sci. Eng. 2007, 9, 90-95. [CrossRef]

44. Hatalis, K. LehighDSM Python Package. 2019. Available online: https://github.com/hatalis/ DSM (accessed on 1 August 2020).

(C) 2020 by the authors. Licensee MDPI, Basel, Switzerland. This article is an open access article distributed under the terms and conditions of the Creative Commons Attribution (CC BY) license (http://creativecommons.org/licenses/by/4.0/). 